\title{
Consensus over Random Graph Processes: Network Borel-Cantelli Lemmas for Almost Sure Convergence
}

\author{
Guodong Shi, Brian D. O. Anderson, and Karl Henrik Johansson
}

\begin{abstract}
Distributed consensus computation over random graph processes is considered. The random graph process is defined as a sequence of random variables which take values from the set of all possible digraphs over the node set. At each time step, every node updates its state based on a Bernoulli trial, independent in time and among different nodes: either averaging among the neighbor set generated by the random graph, or sticking with its current state. Connectivity-independence and arc-independence are introduced to capture the fundamental influence of the random graphs on the consensus convergence. Necessary and/or sufficient conditions are presented on the success probabilities of the Bernoulli trials for the network to reach a global almost sure consensus, with some sharp threshold established revealing a consensus zero-one law. Convergence rates are established by lower and upper bounds of the $\epsilon$ computation time. We also generalize the concepts of connectivity/arc independence to their analogues from the $*$-mixing point of view, so that our results apply to a very wide class of graphical models, including the majority of random graph models in the literature, e.g., Erdős-Rényi, gossiping, and Markovian random graphs. We show that under $*$-mixing, our convergence analysis continues to hold and the corresponding almost sure consensus conditions are established. Finally, we further investigate almost sure finite-time convergence of random gossiping algorithms, and prove that the Bernoulli trials play a key role in ensuring finite-time convergence. These results add to the understanding of the interplay between random graphs, random computations, and convergence probability for distributed information processing.
\end{abstract}

Keywords: Consensus algorithms, Random graphs, Zero-One law, Gossiping

\section{Introduction}

In recent years, there has been considerable research interest on distributed algorithms for information exchange, estimation, and computation over networks. Such algorithms have a variety of potential applications in sensor networks, peer-to-peer networks, wireless networks and networked control systems. 
Targeting the design of simple decentralized algorithms for computation or estimation, where nodes exchange information only with their neighbors, a distributed consensus algorithm serves as a primitive towards more sophisticated information processing algorithms, e.g., [1, 2, 3, 4,

The investigation of the consensus problem has a long history in several scientific fields including computer science [5, 6], engineering [7, 8, and social science [9, 10, 11]. Deterministic consensus algorithms have been extensively studied for both time-invariant and time-varying communication graphs in the literature, with efforts typically devoted to finding proper connectivity conditions that can ensure a desired collective convergence [12, 13, 14, 15. These efforts can indeed be traced back to the study of ergodicity of non-homogeneous Markov chains [16, 17] and distributed decision making [18. In addition, researchers were also interested in the design of weighted averaging algorithms to reach a faster consensus, or to reach a consensus with asynchronous computations [19, 20].

On the other hand, the theory of random graphs [36] is fundamental for the study of in-network communication, information processing, and opinion aggregation, e.g., [37, 39, 10]. Consensus algorithms that are carried out over a random graph process have also drawn attention. In [21], the authors studied linear consensus dynamics with communication graphs defined as a sequence of independent, identically distributed (i.i.d.) Erdős-Rényi random graphs, and almost sure convergence was shown. Then in [22], the analysis was generalized to directed Erdős-Rényi graphs. Mean-square performance for consensus algorithms over i.i.d. random graphs was studied in [23, and the influence of random packet drop was investigated in [24]. In [25], a necessary and sufficient condition was presented on almost sure asymptotic consensus for i.i.d. models. In [26, 27, 28], the authors studied distributed average consensus in sensor networks with quantized data and random link failures. In [29], the communication graph was described as a finite-state Markov chain where each graph corresponds to one state of the chain, and almost sure consensus was concluded by investigating the connectivity of the closed positive recurrent sets of the Markovian random graph. In [30], convergence to consensus was studied under more general linear consensus algorithms, where the random update and control matrices were determined by possibly non-stationary stochastic matrix processes coupled with disturbances. In [31], a general model was investigated for consensus computations over stationary random graph processes with a necessary and sufficient condition presented on the expected graphs regarding almost sure consensus. It is worth emphasizing that besides convergence to a (not necessarily average) consensus, the focus of [23] (and later [10]) was on the error with respect to the average of the initial values, which is far more challenging.

Classical random graph theory suggests that many important properties of static random graphs appear suddenly as some parameter is smoothly adjusted from a probabilistic point of view [36]. To be precise, there exists usually a function, called a threshold, of a parameter (e.g., the graph size), and a quantity 
(e.g., probability of edge appearance) such that a given behaviour appears asymptotically in the parameter if and only if such a quantity grows faster than the threshold. The phenomenon is called a zero-one law. Pioneered in [38, these sharp probabilistic phase transitions have been a central motif in the study of random graphs [39, 40, 41, 42]. In fact, as early as in [25], a zero-one law has been shown to exist for consensus convergence over i.i.d. random graph processes. Naturally, one may wonder, with possible unwillingness or inability for a node to update, would there be any threshold condition which leads to a similar zero-one law with respect to collective convergence of consensus dynamics over random graphs? Although various results have been established to guarantee probabilistic consensus [21, 22, 23, 24, 30, the literature still lacks a general model and analysis for consensus dynamics over random graphs which can accurately describe the fundamental influence of graph processes and whether such a zero-one law could arise.

To this end, in this paper, we study the almost sure convergence of a randomized consensus algorithm over random graph processes. A general random graph process is adopted to model node interactions as a sequence of random variables which take values from the set of all possible graphs with the given node set. Another random node update process is independently built upon this random graph process, in that at each time step, every node independently updates its state as a weighted average of its neighbors' states or sticks with its current state. The choice is a Bernoulli trial with success probability $P_{k}$. We introduce connectivity-independence and arc-independence for the considered random graph processes. For connectivity-independent graphs, we show that $\sum_{k} P_{k}^{n-1}=\infty$ is a sufficient condition for almost sure consensus, where $n$ is the number of nodes. For arc-independent graphs, we show that $\sum_{k} P_{k}=\infty$ is a sharp threshold, i.e., the consensus probability is zero for almost all initial conditions when the sum converges, while it is one for all initial conditions when the sum diverges. In other words, a zero-one law is established for the presented randomized consensus processing over arc-independent graphs, and we see that the success probability of the node updates defines the parameter for the corresponding threshold function. Hence, also consensus computations over random graph processes show the sudden transition similar to Erdős-Rényi graphs, and other models in the literature [36].

\subsection{Problem Definition}

A directed graph (digraph) over $\mathrm{V}$ is defined as $\mathcal{G}=(\mathrm{V}, \mathcal{E})$, where nodes are indexed in the set $\mathrm{V}=$ $\{1,2, \ldots, n\}$ and $\mathcal{E}$ is the set of arcs, i.e., ordered pairs of distinct nodes [33. An arc from $i$ to $j$ is denoted as $(i, j)$. There are $2^{n(n-1)}$ different digraphs with node set V. We label these graphs from 1 to $2^{n(n-1)}$ by an arbitrary order. In the following, we will identify an integer in $\mathscr{G}=\left\{1, \ldots, 2^{n(n-1)}\right\}$ with the corresponding graph in this order. Time is slotted at $k=0,1, \ldots$ We denote by $\mathrm{G}=(\mathrm{V}, \mathrm{E})$ a random 
graph, which by definition is a random variable (over some underlying probability space) that takes value in $\mathscr{G}$.

Let $\mathrm{G}_{k}=\left(\mathrm{V}, \mathrm{E}_{k}\right), k=0,1, \ldots$ be a sequence of random graphs over node set $\mathrm{V}$. We call node $j$ a neighbor of $i$ at time $k$ if $(j, i) \in \mathrm{G}_{k}$. Each node is supposed to always be a neighbor of itself. Denote $\mathrm{N}_{i}(k)$ as the set of neighbors of node $i$ at time $k$. Each node $i$ holds a state $x_{i}(k) \in \mathbb{R}$ at time $k$. Let $\left\langle P_{k}\right\rangle$ be a given deterministic sequence with $0<P_{k}<1$ for all $k$. Independent of the graph process, node states, and other nodes, the updating rule of node $x_{i}(k)$ is as follows:

$$
x_{i}(k+1)= \begin{cases}\sum_{j \in \mathrm{N}_{i}(k)} a_{i j}(k) x_{j}(k), & \text { with probability } P_{k} ; \\ x_{i}(k), & \text { with probability } 1-P_{k} .\end{cases}
$$

Here $a_{i j}(k)$ represents the weight of arc $(j, i)$. We use the following weights rule as our standing assumption, cf., [1, 15, 43].

Assumption (Weights Rule) (i) $\sum_{j \in \mathrm{N}_{i}(k)} a_{i j}(k)=1$ for all $i$ and $k$; (ii) there exists a constant $\eta>0$ such that $a_{i j}(k) \geq \eta$ for all $i, j$ and $k$.

Algorithm (1) can also be written as the following dynamics:

$$
x_{i}(k+1)=\chi_{i}(k)\left(\sum_{j \in \mathrm{N}_{i}(k)} a_{i j}(k) x_{j}(k)\right)+\left(1-\chi_{i}(k)\right) x_{i}(k)
$$

where $\chi_{i}(k), i=1, \ldots, n, k \in \mathbb{N}$ are independent Bernoulli random variables with $\mathbf{E}\left[\chi_{i}(k)\right]=P_{k}$ for all $i$, which are also independent with $\left\langle\mathrm{G}_{k}\right\rangle$.

The randomized information processing (1) describes the possible unwillingness or inability of a node to update, even if it receives information from other nodes. For instance, in the opinion dynamics of social networks, forceful belief exchanges may happen randomly by Bernoulli decisions for individuals so that misinformation may be spread [10]. From an engineering viewpoint, in wireless communication nodes may be asleep or broken randomly due to the unpredictability of the environment and the unreliability of the networked communication [23, 27]. The above standing assumption is adopted throughout the paper without specific mentioning. We note that in order to satisfy the standing assumption, the $a_{i j}(k)$ can be either deterministic or random, e.g., $a_{i j}(k)=1 / n$ for $j \neq i \in \mathrm{N}_{i}(k)$ and $a_{i i}(k)=1-\left|\mathrm{N}_{i}(k)\right| / n$; or $a_{i j}(k)=1 /\left|\mathrm{N}_{i}(k)\right|$ for all $j \in \mathrm{N}_{i}(k)$.

Our interest is in the convergence of the randomized consensus algorithm and the time it takes for the network to reach a consensus. Let $x(k)=\left(x_{1}(k) \ldots x_{n}(k)\right)^{\mathrm{T}} \in \mathbb{R}^{n}$ be the random sequence driven by the randomized algorithm (11) for initial condition $x^{0}=\left(x_{1}(0) \ldots x_{n}(0)\right)^{\mathrm{T}}$. Denote

$$
H(k):=\max _{i \in \mathrm{V}}\left\{x_{i}(k)\right\}, \quad h(k):=\min _{i \in \mathrm{V}}\left\{x_{i}(k)\right\}
$$


as the maximal and minimal states among all nodes, respectively, and define $\mathcal{H}(k):=H(k)-h(k)$ as a consensus measure. Let $\mathbf{P}$ be the probability measure capturing all the randomness in $\langle x(k)\rangle$, and whenever necessary we write $\mathbf{P}_{x^{0}}$ indicating the probability measure for initial value $x^{0}$.

Under our standing assumption there always holds that $\mathcal{H}(k+1) \leq \mathcal{H}(k)$. We introduce the following definition.

Definition 1 Global almost sure (a.s.) consensus is achieved for Algorithm (1) if $\mathbf{P}\left(\lim _{k \rightarrow \infty} \mathcal{H}(k)=\right.$ $0)=1$ for all $x^{0} \in \mathbb{R}^{n}$. Moreover, for any $0<\epsilon<1$, the $\epsilon$-computation time is defined as

$$
\mathscr{T}_{\mathrm{com}}(\epsilon) \doteq \sup _{x^{0} \in \mathbb{R}^{n}} \inf \{k: \quad \mathbf{P}(\mathcal{H}(k) \geq \epsilon \mathcal{H}(0)) \leq \epsilon\}
$$

\subsection{Main Results}

Let $x^{0}$ be considered under the standard Lebesgue measure over $\mathbb{R}^{n}$. We first present the following result on the impossibility of consensus, which is built for arbitrary random graph process $\left\langle\mathrm{G}_{k}\right\rangle$.

Theorem 1 (i). Global a.s. consensus can be achieved for Algorithm (1) only if $\sum_{k=0}^{\infty} P_{k}=\infty$, and a universal lower bound for $\mathscr{T}_{\mathrm{com}}(\epsilon)$ is given by

$$
\mathscr{T}_{\text {com }}(\epsilon) \geq \sup \left\{k: \quad \sum_{i=0}^{k-1} \log \left(1-P_{i}\right)^{-1} \leq \frac{\log \epsilon^{-1}}{2}\right\} .
$$

(ii). Suppose $\sum_{k=0}^{\infty} P_{k}<\infty$ and $\left\{a_{i j}(k): i, j \in \mathrm{V}, k \in \mathbb{N}\right\}$ contains at most countably many elements. Then one of the following must happen:

a) there exists a constant $p^{b}>0$ such that $\mathbf{P}_{x^{0}}\left(\lim _{k \rightarrow \infty} \mathcal{H}(k)=0\right) \geq p^{b}$ for all $x^{0} \in \mathbb{R}^{n}$.

b) $\mathbf{P}_{x^{0}}\left(\lim _{k \rightarrow \infty} \mathcal{H}(k)=0\right)=0$ for almost all $x^{0} \in \mathbb{R}^{n}$.

(iii). Suppose $\sum_{k=0}^{\infty} P_{k}<\infty$ and there exists a constant $a_{*}$ such that $a_{i i}(k) \geq a_{*}>1 / 2$ for all $i$ and $k$. Then $\mathbf{P}\left(\lim _{k \rightarrow \infty} \mathcal{H}(k)=0\right)=0$ for almost all $x^{0} \in \mathbb{R}^{n}$.

Recall that a digraph $\mathrm{G}$ is said to be quasi-strongly connected if $\mathrm{G}$ has a directed spanning tree [34]. We introduce the following definition.

Definition 2 The random graph process $\left\langle\mathrm{G}_{k}\right\rangle$ is called connectivity-independent if the events

$$
\left\{\mathrm{G}_{k} \text { is quasi-strongly connected }\right\}, k \in \mathbb{N}
$$

are mutually independent.

For connectivity-independent graphs, we present the following almost sure convergence result. 
Theorem 2 Suppose $\left\langle\mathrm{G}_{k}\right\rangle$ is connectivity-independent and there exists a constant $0<q<1$ such that $\mathbf{P}\left(\mathrm{G}_{k}\right.$ is quasi-strongly connected $) \geq q$ for all $k$. Assume in addition that $P_{k+1} \leq P_{k}$. Then Algorithm (1) achieves global a.s. consensus if $\sum_{k=0}^{\infty} P_{k}^{n-1}=\infty$, and an upper bound of $\mathscr{T}_{\text {com }}(\epsilon)$ is given as

$$
\mathscr{T}_{\text {com }}(\epsilon) \leq \inf \left\{M: \quad \sum_{i=0}^{M-1} \log \left(1-\frac{(q \eta)^{(n-1)^{2}}}{2} \cdot P_{(i+1)(n-1)^{2}}^{n-1}\right)^{-1} \geq \log \epsilon^{-2}\right\} \times(n-1)^{2} .
$$

We believe that the convergence condition given in Theorem 2 is reasonably tight since the probability that all the arcs in Algorithm (1) are active at time $k$ is $P_{k}^{n}$, while one single inactive arc may be enough to break the connectivity of the graph (note that even the condition $\sum_{k=0}^{\infty} P_{k}^{n-1}=\infty$ allows $P_{k}$ to decrease to zero). The condition $P_{k+1} \leq P_{k}$ is a technical assumption which enforces certain regularity of the node update frequencies 1 . While the sufficient convergence condition $\sum_{k=0}^{\infty} P_{k}^{n-1}=\infty$ indicates it is more difficult to reach consensus over large networks.

Nevertheless, connectivity is a global property of a graph, and indeed it does not rely on any specific arc. The next definition is on the independence of the existence of the arcs in the graph process.

Definition 3 Let $\mathcal{G}^{\dagger}=\left(\mathrm{V}, \mathcal{E}^{\dagger}\right)$ be a (deterministic) digraph with $\mathcal{E}^{\dagger}=\left\{\left(i_{\tau}, j_{\tau}\right): \tau=1, \ldots,\left|\mathcal{E}^{\dagger}\right|\right\}$. Denote $\mathcal{E}_{k}(\tau):=\left\{\left(i_{\tau}, j_{\tau}\right) \in \mathrm{G}_{k}\right\}$ for $\tau=1, \ldots,\left|\mathcal{E}^{\dagger}\right|$ and $k \in \mathbb{N}$. Then $\left\langle\mathrm{G}_{k}\right\rangle$ is called arc-independent with respect to $\mathcal{G}^{\dagger}$ if the events

$$
\mathcal{E}_{k}\left(\tau_{k}\right), k \in \mathbb{N}
$$

are mutually independent for any deterministic sequence $\left\langle\tau_{k}\right\rangle$ with each $\tau_{k}$ taking value from $\left\{1, \ldots,\left|\mathcal{E}^{\dagger}\right|\right\}$. We call $\mathcal{G}^{\dagger}$ a basic graph of the random graph process $\left\langle\mathrm{G}_{k}\right\rangle$.

For arc-independent graphs, the following result holds.

Theorem 3 Suppose $\left\langle\mathrm{G}_{k}\right\rangle$ is arc-independent with respect to the basic graph $\mathcal{G}^{\dagger}=\left(\mathrm{V}, \mathcal{E}^{\dagger}\right)$. Let $\mathcal{G}^{\dagger}$ be quasi-strongly connected and assume that there exists a constant $0<q_{*}<1$ such that $\mathbf{P}\left((i, j) \in \mathrm{E}_{k}\right) \geq q_{*}$ for all $k$ and $(i, j) \in \mathcal{E}^{\dagger}$. Then Algorithm (1) achieves global a.s. consensus if and only if $\sum_{k=0}^{\infty} P_{k}=\infty$, and there holds

$$
\mathscr{T}_{\text {com }}(\epsilon) \leq \inf \left\{k: \sum_{i=0}^{k-1}\left(1-\left(1-P_{i}\right)^{n}\right) \geq \frac{(n-1)\left|\mathcal{E}^{\dagger}\right|}{\log A} \log \left(A \epsilon^{2} / n\right)\right\}
$$

where $A=1-\left(\eta q_{*} / n\right)^{(n-1)\left|\mathcal{E}^{\dagger}\right|}$ and $\left|\mathcal{E}^{\dagger}\right|$ represents the number of elements in $\mathcal{E}^{\dagger}$.

\footnotetext{
${ }^{1}$ The condition $P_{k+1} \leq P_{k}$ is also to make the problem more interesting since the main challenge is to reach consensus with as small $P_{k}$ as possible. Certainly the counter condition $P_{k+1} \geq P_{k}$ will ensure convergence straightforwardly.
} 


\subsection{Remarks}

Theorems 1 and 3 combined show that $\sum_{k=0}^{\infty} P_{k}=\infty$ is a sharp threshold for Algorithm (1) to reach consensus convergence under quite general conditions leading to a Zero-One Law: consensus convergence holds true with probability one for all initial conditions when the infinite sum diverges, and otherwise consensus is achieved with probability zero for almost all initial conditions. These results are essentially due to the Borel-Cantelli Lemma, where the condition $\sum_{k=0}^{\infty} P_{k}=\infty$ ensures that a.s. every node updates its states infinitely often. Consensus convergence however requires much more than that: it is crucial that every node updates infinitely often (cf., Theorem 1), but more importantly, these node updates should essentially comply with the underlying random graph process (cf., Theorems 2 and 3 ).

The convergence time estimates given in Theorems 2 and 3 are established with the help of Markov's inequality, where the random graph process and the Bernoulli trials together cumulatively contribute to the upper bound. Moreover, from the proofs it is straightforward to see that all the results can be easily generalized to the case when nodes have distinct successful update probabilities, i.e., the $P_{k}$ become $P_{k}^{i}$ depending on $i \in \mathrm{V}$. For the ease of presentation we let $\left\langle P_{k}\right\rangle$ apply to all nodes.

The proposed concepts of connectivity and arc independence directly apply to many random graph models in the literature, for which a detailed discussion is provided in Subection 4.1 illustrating the usefulness of the derived results for several fundamental random graph processes. In fact, we manage to generalize the connectivity/arc independence to connectivity/arc mixing of random graph processes, and show that the same analysis smoothly carries on leading to similar convergence results. As a result, the majority of the random graph models in the literature (including Markovian random graphs) are then covered. The generalization to mixing of random graphs is in Subsection 4.2 ,

Finally, for random gossiping algorithms [2], we are able to go one step forward and present almost sure finite-time convergence results. Remarkably enough, the random node update process driven by nonreliable $\left\langle P_{k}\right\rangle$ becomes essential for almost sure finite-time convergence. This part of the generalization is in Subsection 4.3 .

\subsection{Paper Organization}

The remainder of the paper is organized as follows. Section 2 establishes the convergence results for connectivity-independent graphs. Several classes of connectivity-independent graphs, e.g., directed, bidirectional and acyclic graphs, are investigated, respectively. The proof of Theorem 2 is therefore obtained as a direct consequence. Section 3 turns to arc-independent graphs and proves Theorem 3 using a matrix argument. Section 4 is devoted to some nontrivial generalizations of the main results: A few basic random graph models in the literature are discussed illustrating the applicability of the derived results; the connec- 
tivity/arc $*$-mixing random graphs are introduced with the corresponding convergence results established; conditions for almost sure finite-time convergence of random gossiping algorithms are presented. Finally some concluding remarks are given in Section 5 ,

The proof of Theorem 1 on the consensus impossibilities is a bit tangential to the a.s. convergence results, and is therefore put in Appendix A.

\section{Notation and Terminologies}

A (simple) directed graph $\mathrm{G}=(\mathrm{V}, \mathrm{E})$, or in short, a digraph, consists of a finite set $\mathrm{V}=\{1, \ldots, N\}$ of nodes and an arc set $\mathrm{E}$, where an element $e=(i, j) \in \mathrm{E}$ denotes an arc from node $i \in \mathrm{V}$ to $j \in \mathrm{V}$ with $i \neq j$. A directed path between two vertices $v_{1}$ and $v_{k}$ in $\mathrm{G}$ is a sequence of distinct nodes $v_{1} v_{2} \ldots v_{k}$ such that for any $m=1, \ldots, k-1$, there is an arc from $v_{m}$ to $v_{m+1} ; v_{1} v_{2} \ldots v_{k}$ is called a semi-path if for any $m=1, \ldots, k-1$, either $\left(v_{m}, v_{m+1}\right) \in \mathrm{E}$ or $\left(v_{m+1}, v_{m}\right) \in \mathrm{E}$. As is usual [33], a graph $\mathrm{G}$ is termed strongly connected if, for every pair of distinct nodes in $\mathrm{V}$, there is a path from one to the other; quasi-strongly connected if there exists a node $v \in \mathcal{V}$, namely a root, such that there is a path from $v$ to all other nodes (equivalently, the graph contains a directed spanning tree); weakly connected if there is a semi-path between any two distinct nodes. Deterministic graphs are denoted by $\mathcal{G}$ with arc set $\mathcal{E}$; random graphs are denoted by $\mathrm{G}$ with arc set $\mathrm{E}$.

We use $\mathbb{N}$ to denote the set of non-negative integers, and $\mathbb{R}$ denotes real numbers. Probability is denoted as $\mathbf{P}(\cdot)$; expectation of random variables is denoted as $\mathbf{E}[\cdot]$; events are denoted by $\mathcal{A}, \mathcal{B}, \ldots$ We also use $|\cdot|$ to represent the cardinality of a finite set, or the absolute value of a real number. A sequence $\left\{b_{k}\right\}_{k=0}^{\infty}$ of real numbers, random variables, or events, is always abbreviated as $\left\langle b_{k}\right\rangle$.

\section{Connectivity-Independent Graphs}

In this section, we present the convergence analysis for connectivity-independent random graph processes. We are going to study some general cases relying on the joint graphs only.

The joint graph [18, 1] of $\mathrm{G}_{k}$ on time interval $\left[k_{1}, k_{2}\right]$ for $0 \leq k_{1} \leq k_{2} \leq \infty$ is defined as $\mathrm{G}\left(\left[k_{1}, k_{2}\right]\right)=$ $\left(\mathrm{V}, \bigcup_{k \in\left[k_{1}, k_{2}\right]} \mathrm{E}_{k}\right)$. We introduce the following definition of connectivity for the random graph process.

Definition 4 The random graph process $\left\langle\mathrm{G}_{k}\right\rangle$ is termed

(i) uniformly stochastically quasi-strongly connected, if there exist an integer $B \geq 1$ and $0<q<1$ such that the sequence $\langle\mathrm{G}([m B,(m+1) B-1])\rangle$ is connectivity-independent and

$$
\mathbf{P}(\mathrm{G}([m B,(m+1) B-1]) \text { is quasi-strongly connected }) \geq q, \quad m=0,1, \ldots ;
$$


(ii) infinitely stochastically quasi-strongly connected, if there exist a (deterministic) sequence $0=C_{0}<$ $\cdots<C_{m}<\ldots$ and a constant $0<q<1$ such that $\left\langle\mathrm{G}\left(\left[C_{m}, C_{m+1}\right)\right)\right\rangle$ is connectivity-independent and

$$
\mathbf{P}\left(\mathrm{G}\left(\left[C_{m}, C_{m+1}\right)\right) \text { is quasi-strongly connected }\right) \geq q, \quad m=0,1, \ldots
$$

In the remainder of this section, we first establish consensus conditions for uniformly stochastically quasi-strongly connected graph processes, and the proof of Theorem 2 is obtained as a special case. Next, two special cases, bidirectional and acyclic graph processes, are further investigated, respectively.

\subsection{Uniform Stochastic Connectivity}

The following result is for consensus convergence over uniformly stochastically quasi-strongly connected graphs.

Proposition 1 Suppose $\left\langle\mathrm{G}_{k}\right\rangle$ is uniformly stochastically quasi-strongly connected with $B \geq 1$ and $q>0$. Then Algorithm (1) achieves global a.s. consensus if $\sum_{s=0}^{\infty} \bar{P}_{s}=\infty$, where

$$
\bar{P}_{s}=\inf _{\alpha_{1}<\cdots<\alpha_{n-1}}\left\{\prod_{l=1}^{n-1} P_{\alpha_{l}}: \alpha_{l} \in\left[s(n-1)^{2} B,(s+1)(n-1)^{2} B\right), l=1, \ldots, n-1\right\} .
$$

Moreover, we have

$$
\mathscr{T}_{\text {com }}(\epsilon) \leq \inf \left\{M: \quad \sum_{i=0}^{M-1} \log \left(1-\frac{(q \eta / n)^{(n-1)^{2}}}{2} \cdot \bar{P}_{i}\right)^{-1} \geq \log \epsilon^{-2}\right\} \times(n-1)^{2} B,
$$

where $\eta$ is the constant defined in the weights rule.

Proof. We first establish a lemma characterizing a useful property of uniformly stochastically quasi-strongly connected graphs.

Lemma 1 Assume that $\left\langle\mathrm{G}_{k}\right\rangle$ is uniformly stochastically quasi-strongly connected. Then for any $s=$ $0,1, \ldots$, we have

$$
\begin{aligned}
\mathbf{P}\left(\exists i_{0} \in \mathrm{V} \text { and } \tau_{1}<\cdots<\tau_{n-1} \in\left[s(n-1)^{2},(s+1)(n-1)^{2}\right)\right. \\
\text { s.t. } \left.i_{0} \text { is a root of } \mathrm{G}\left(\left[\tau_{j} B,\left(\tau_{j}+1\right) B-1\right]\right) \text { for all } j=1, \ldots, n-1\right) \geq\left(\frac{q}{n}\right)^{(n-1)^{2}} .
\end{aligned}
$$

Proof. The probability that the graph $\mathrm{G}([\tau B,(\tau+1) B-1])$ has a root is no less than $q$ for $\tau=s(n-$ $1)^{2}, \ldots,(s+1)(n-1)^{2}-1$. Consequently, for each $\mathrm{G}([\tau B,(\tau+1) B-1])$, there is a (deterministic) node $i_{0}$ (which depends on $\tau$ ) such that $i_{0}$ is a root with probability at least $q / n$. The $(n-1)^{2}$ graphs, $\mathrm{G}([\tau B,(\tau+1) B-1]), \tau=s(n-1)^{2}, \ldots,(s+1)(n-1)^{2}-1$ will lead to at least $(n-1)^{2}$ such roots (with possible repetitions, of course). However, the total number of the nodes is $n$. Thus, at least one 
node is counted more than $n-2$ times. Then the desired lemma immediately follows from the definition of connectivity independence.

Denote $k_{s}=s(n-1)^{2} B$ for $s \geq 0$. We consider the event studied in Lemma1, that there exists a common root node $i_{0}$ such that $i_{0}$ is a root of $\mathrm{G}\left(\left[\tau_{j} B,\left(\tau_{j}+1\right) B-1\right]\right)$ for $j=1, \ldots, n-1$ with $k_{s} \leq \tau_{j} B \leq k_{s+1}-1$ with a probability no smaller than $(q / n)^{(n-1)^{2}}$. We first assume that

$$
x_{i_{0}}\left(k_{s}\right) \leq \frac{1}{2} h\left(k_{s}\right)+\frac{1}{2} H\left(k_{s}\right) .
$$

From a symmetric analysis by establishing the lower bound of $h\left(k_{s+1}\right)$ (or, directly considering $y(k)=$ $\left(y_{1}(k) \ldots y_{n}(k)\right)^{\mathrm{T}}$ with $\left.y_{i}(k)=-x_{i}(k)\right)$, it is easy to show that the argument continues to hold under the condition that

$$
x_{i_{0}}\left(k_{s}\right)>\frac{1}{2} h\left(k_{s}\right)+\frac{1}{2} H\left(k_{s}\right) .
$$

We divide the rest of the proof into four steps.

Step 1 . In this step, we bound $x_{i_{0}}(k)$. With the weights rule in our standing assumption, we have

$$
\begin{aligned}
\sum_{j \in \mathrm{N}_{i_{0}}\left(k_{s}\right)} a_{i_{0} j}\left(k_{s}\right) x_{j}\left(k_{s}\right) & =a_{i_{0} i_{0}}\left(k_{s}\right) x_{i_{0}}\left(k_{s}\right)+\sum_{j \in \mathrm{N}_{i_{0}}\left(k_{s}\right) \backslash\left\{i_{0}\right\}} a_{i_{0} j}\left(k_{s}\right) x_{j}\left(k_{s}\right) \\
& \leq a_{i_{0} i_{0}}\left(k_{s}\right)\left(\frac{1}{2} h\left(k_{s}\right)+\frac{1}{2} H\left(k_{s}\right)\right)+\left(1-a_{i_{0} i_{0}}\left(k_{s}\right)\right) H\left(k_{s}\right) \\
& =\frac{a_{i_{0} i_{0}}\left(k_{s}\right)}{2} h\left(k_{s}\right)+\left(1-\frac{a_{i_{0} i_{0}}\left(k_{s}\right)}{2}\right) H\left(k_{s}\right) \\
& \leq \frac{\eta}{2} h\left(k_{s}\right)+\left(1-\frac{\eta}{2}\right) H\left(k_{s}\right)
\end{aligned}
$$

where the last equality holds from the facts that $h\left(k_{s}\right) \leq H\left(k_{s}\right)$ and $a_{i_{0} i_{0}}\left(k_{s}\right) \geq \eta$. Thus, since $\eta<1$, no matter whether node $i_{0}$ takes averaging or sticks to its current state, we obtain

$$
x_{i_{0}}\left(k_{s}+1\right) \leq \frac{\eta}{2} h\left(k_{s}\right)+\left(1-\frac{\eta}{2}\right) H\left(k_{s}\right) .
$$

By recursively applying (4) there holds that for any $\varrho=0,1, \ldots$,

$$
x_{i_{0}}\left(k_{s}+\varrho\right) \leq \frac{\eta^{\varrho}}{2} h\left(k_{s}\right)+\left(1-\frac{\eta^{\varrho}}{2}\right) H\left(k_{s}\right) .
$$

Step 2. Conditioned that $i_{0}$ is a root of $\mathrm{G}\left(\left[\tau_{1} B,\left(\tau_{1}+1\right) B-1\right]\right)$, there will be $i_{1} \in \mathrm{V}$, which is different from $i_{0}$, and a time instant $t_{1} \in\left[\tau_{1} B,\left(\tau_{1}+1\right) B-1\right]$, such that $\left(i_{0}, i_{1}\right) \in \mathrm{E}_{t_{1}}$. Suppose $t_{1}=k_{s}+\varsigma$. In this step, we bound $x_{i_{1}}(k)$.

\footnotetext{
${ }^{2}$ It should be emphasized that here both $i_{1}$ and $t_{1}$ could be random. We can however treat the remaining analysis for each sample path $\omega$ of $i_{1}(\omega)$ and $t_{1}(\omega)$, and the same conclusion will be drawn. Therefore, without loss of generality, we omit the discussion regarding possible randomness in $i_{1}$ and $t_{1}$.
} 
If $i_{1}$ takes an averaging update at time step $t_{1}$, we obtain from (51) that

$$
\begin{aligned}
x_{i_{1}}\left(k_{s}+\varsigma+1\right) & =a_{i_{1} i_{0}}\left(k_{s}+\varsigma\right) x_{i_{0}}\left(k_{s}+\varsigma\right)+\sum_{j \in \mathrm{N}_{i_{1}}\left(k_{s}+\varsigma\right) \backslash\left\{i_{0}\right\}} a_{i_{1} j}\left(k_{s}+\varsigma\right) x_{j}\left(k_{s}+\varsigma\right) \\
& \leq \eta\left[\frac{\eta^{\varsigma}}{2} h\left(k_{s}\right)+\left(1-\frac{\eta^{\varsigma}}{2}\right) H\left(k_{s}\right)\right]+(1-\eta) H\left(k_{s}\right) \\
& =\frac{\eta^{\varsigma+1}}{2} h\left(k_{s}\right)+\left(1-\frac{\eta^{\varsigma+1}}{2}\right) H\left(k_{s}\right)
\end{aligned}
$$

which leads to that for any $\varrho=\left(\tau_{1}+1\right) B-k_{s}, \ldots$,

$$
x_{i_{1}}\left(k_{s}+\varrho\right) \leq \frac{\eta^{\varrho}}{2} h\left(k_{s}\right)+\left(1-\frac{\eta^{\varrho}}{2}\right) H\left(k_{s}\right) .
$$

Therefore, we conclude from (5) and (77) that

$$
\mathbf{P}\left(x_{i_{l}}\left(k_{s}+\varrho\right) \leq \frac{\eta^{\varrho}}{2} h\left(k_{s}\right)+\left(1-\frac{\eta^{\varrho}}{2}\right) H\left(k_{s}\right), l=0,1 ; \varrho \geq\left(\tau_{1}+1\right) B-k_{s}\right) \geq\left(\frac{q}{n}\right)^{(n-1)^{2}} \min _{k \in\left[\tau_{1} B,\left(\tau_{1}+1\right) B-1\right]} P_{k} .
$$

Step 3. We proceed the analysis for the time interval $\left[\tau_{2} B,\left(\tau_{2}+1\right) B-1\right]$. Similarly, $i_{2} \neq i_{0}, i_{1}$ can be found (because $i_{0}$ is a root) such that

$$
\begin{array}{r}
\mathbf{P}\left(x_{i_{l}}\left(k_{s}+\varrho\right) \leq \frac{\eta^{\varrho}}{2} h\left(k_{s}\right)+\left(1-\frac{\eta^{\varrho}}{2}\right) H\left(k_{s}\right), l=0,1,2 ; \varrho \geq\left(\tau_{2}+1\right) B-k_{s}\right) \\
\geq\left(\frac{q}{n}\right)^{(n-1)^{2}} \min _{\alpha_{1}, \alpha_{2} \in\left[\tau_{1} B,\left(\tau_{2}+1\right) B-1\right]} P_{\alpha_{1}} P_{\alpha_{2}} .
\end{array}
$$

Continuing for time intervals $\left[\tau_{j} B,\left(\tau_{j}+1\right) B-1\right]$ for $j=3, \ldots, n-1$, bounds for $i_{3}, \ldots, i_{n-1}$ can be established as

$\mathbf{P}\left(x_{i_{m}}\left(k_{s}+\varrho\right) \leq \frac{\eta^{\varrho}}{2} h\left(k_{s}\right)+\left(1-\frac{\eta^{\varrho}}{2}\right) H\left(k_{s}\right), m=0, \ldots, n-1 ; \varrho=\left(\tau_{n-1}+1\right) B-k_{s}, \ldots\right) \geq \bar{P}_{s}\left(\frac{q}{n}\right)^{(n-1)^{2}}$,

with

$$
\bar{P}_{s}=\inf _{\alpha_{1}<\cdots<\alpha_{n-1}}\left\{\prod_{m=1}^{n-1} P_{\alpha_{m}}: \alpha_{m} \in\left[s(n-1)^{2} B,(s+1)(n-1)^{2} B\right), m=1, \ldots, n-1\right\} .
$$

This leads to

$$
\mathbf{P}\left(H\left(k_{s+1}\right) \leq \frac{\eta^{(n-1)^{2}}}{2} h\left(k_{s}\right)+\left(1-\frac{\eta^{(n-1)^{2}}}{2}\right) H\left(k_{s}\right)\right) \geq \bar{P}_{s}\left(\frac{q}{n}\right)^{(n-1)^{2}},
$$

which implies

$$
\mathbf{P}\left(\mathcal{H}\left(k_{s+1}\right) \leq\left(1-\frac{\eta^{(n-1)^{2}}}{2}\right) \mathcal{H}\left(k_{s}\right)\right) \geq \bar{P}_{s}\left(\frac{q}{n}\right)^{(n-1)^{2}} .
$$

Therefore, noticing $\mathcal{H}(k+1) \leq \mathcal{H}(k)$ always holds, we obtain from (9) that

$$
\mathbf{E}\left[\mathcal{H}\left(k_{s+1}\right)\right] \leq\left(1-\frac{(q \eta / n)^{(n-1)^{2}}}{2} \cdot \bar{P}_{s}\right) \mathbf{E}\left[\mathcal{H}\left(k_{s}\right)\right]
$$


Step 4. In light of connectivity-independence and the fact that (9) holds only requiring the connectivity of the union graph of $n-1$ subintervals in $\left[s(n-1)^{2},(s+1)(n-1)^{2}\right)$, we further conclude from (10) that 3

$$
\mathbf{E}\left[\mathcal{H}\left(k_{M}\right)\right] \leq \prod_{s=0}^{M-1}\left(1-\frac{(q \eta / n)^{(n-1)^{2}}}{2} \cdot \bar{P}_{s}\right) \mathcal{H}(0)
$$

for all $M \geq 1$. Thus, it follows from Lemma 7 that

$$
\lim _{M \rightarrow \infty} \mathbf{E}\left[\mathcal{H}\left(k_{M}\right)\right]=0
$$

which yields $\lim _{k \rightarrow \infty} \mathbf{E}[\mathcal{H}(k)]=0$ since $\mathcal{H}(k)$ is non-increasing. Using Fatou's Lemma, we further obtain

$$
0 \leq \mathbf{E}\left[\lim _{k \rightarrow \infty} \mathcal{H}(k)\right] \leq \lim _{k \rightarrow \infty} \mathbf{E}[\mathcal{H}(k)]=0
$$

Therefore, the convergence claim of the conclusion holds because (13) implies

$$
\mathbf{P}\left(\lim _{k \rightarrow+\infty} \mathcal{H}(k)=0\right)=1
$$

Applying Markov's Inequality to (11) leads to

$$
\mathbf{P}\left(\mathcal{H}\left(k_{M}\right) \geq \epsilon \mathcal{H}(0)\right) \leq \frac{1}{\epsilon} \cdot \frac{\mathbf{E}\left[\mathcal{H}\left(k_{M}\right)\right]}{\mathcal{H}(0)} \leq \frac{1}{\epsilon} \prod_{s=0}^{M-1}\left(1-\frac{(q \eta / n)^{(n-1)^{2}}}{2} \cdot \bar{P}_{s}\right) .
$$

Consequently, we have

$$
\mathscr{T}_{\text {com }}(\epsilon) \leq \inf \left\{M: \sum_{s=0}^{M-1} \log \left(1-\frac{(q \eta / n)^{(n-1)^{2}}}{2} \cdot \bar{P}_{s}\right)^{-1} \geq \log \epsilon^{-2}\right\} \times(n-1)^{2} B .
$$

The desired conclusion follows.

Remark 1 Suppose $P_{k+1} \leq P_{k}$ for all $k$. Then it is not hard to see that $\sum_{s=0}^{\infty} \bar{P}_{s}=\infty$ if and only if $\sum_{k=0}^{\infty} P_{k}^{n-1}=\infty$. Then Theorem Q holds immediately from Proposition 1 .

\subsection{Bidirectional Connections}

A digraph $\mathcal{G}=(\mathrm{V}, \mathcal{E})$ is bidirectional if for any two nodes $i$ and $j,(i, j) \in \mathcal{E}$ if and only if $(j, i) \in \mathcal{E}$. In this subsection, we study bidirectional graphs.

Note that we do not impose an upper bound for the length of the intervals $\left[C_{m}, C_{m+1}\right)$ in the definition of infinitely stochastically quasi-strong connectivity, which makes an essential difference from the bounded joint connections.

\footnotetext{
${ }^{3}$ Note that in general recursively applying (10) for $s=0,1, \ldots$ requires independence of the random graph process $\left\langle\mathrm{G}_{k}\right\rangle$ that drives $x(k)$. However, from the proof of Step 2 it is clear that connectivity independence has been enough for granting such a recursive analysis and therefore ensuring (11).
} 
Proposition 2 Suppose there holds a.s. that $\mathrm{G}_{k}$ is bidirectional for all $k \geq 0$. Assume that $\left\langle\mathrm{G}_{k}\right\rangle$ is stochastically infinitely quasi-strongly connected. Then Algorithm (1) achieves global a.s. consensus if $\sum_{s=0}^{\infty} \hat{P}_{s}=\infty$, where

$$
\hat{P}_{s}=\inf _{\alpha_{1}<\cdots<\alpha_{n-1}}\left\{\prod_{l=1}^{n-1} P_{\alpha_{l}}: \quad \alpha_{l} \in\left[C_{s(n-1)}, C_{(s+1)(n-1)}\right), l=1, \ldots, n-1\right\} .
$$

and also

$$
\mathscr{T}_{\text {com }}(\epsilon) \leq \inf \left\{C_{s(n-1)}: \sum_{i=0}^{s-1} \log \left(1-(q \eta)^{(n-1)} \cdot \hat{P}_{i}\right)^{-1} \geq \log \epsilon^{-2}\right\} .
$$

Proof. The proof is carried out analyzing a sequence a stopping times (cf., [35]) of the random graph process. Take a node $i_{0} \in \mathrm{V}$ with $x_{i_{0}}\left(C_{0}\right)=h\left(C_{0}\right)$. Define

$$
t_{1}=\inf _{k \geq C_{0}}\left\{\mathrm{~N}_{i_{0}}(k) \backslash\left\{i_{0}\right\} \neq \emptyset\right\}
$$

In other words, $t_{1}$ is the first time that $i_{0}$ has a neighbor other than itself in the random graph process. Then $t_{1}$ is a stopping time of the graph process $\left\langle\mathrm{G}_{k}\right\rangle$, and there must hold

$$
\mathbf{P}\left(t_{1}<C_{1}\right)>q
$$

since $\left\langle\mathrm{G}_{k}\right\rangle$ is infinitely stochastically quasi-strongly connected. We next introduce

$$
\mathrm{V}_{1}=\left\{j \neq i_{0}:\left(i_{0}, j\right) \in \mathrm{E}_{t_{1}}\right\}
$$

We emphasize that by its definition $V_{1}$ is nonempty. Again we proceed in steps for the remaining of the argument.

Step 1. Noticing that $x_{i_{0}}\left(t_{1}\right)=x_{i_{0}}\left(C_{0}\right)$ from the structure of Algorithm (5), we conclude that

$$
\begin{aligned}
x_{i_{0}}\left(t_{1}+1\right) & =\sum_{j \in \mathrm{N}_{i_{0}}\left(t_{1}\right)} a_{i_{0} j}\left(t_{1}\right) x_{j}\left(t_{1}\right) \\
& =a_{i_{0} i_{0}}\left(t_{1}\right) x_{i_{0}}\left(t_{1}\right)+\sum_{j \in \mathrm{N}_{i_{0}}\left(t_{1}\right) \backslash\left\{i_{0}\right\}} a_{i_{0} j}\left(t_{1}\right) x_{j}\left(t_{1}\right) \\
& \leq a_{i_{0} i_{0}}\left(t_{1}\right) h\left(C_{0}\right)+\left(1-a_{i_{0} i_{0}}\left(t_{1}\right)\right) H\left(C_{0}\right) \\
& \leq \eta h\left(C_{0}\right)+(1-\eta) H\left(C_{0}\right)
\end{aligned}
$$

if $i_{0}$ updates its state at time $t_{1}+1$. Note that (14) continues to hold even if $i_{0}$ fails to update at time 
$t_{1}+1$. On the other hand, for $i_{1} \in \mathrm{V}_{1}$, if $i_{q}$ successfully updates its state at time $t_{1}+1$, then

$$
\begin{aligned}
x_{i_{1}}\left(t_{1}+1\right) & =\sum_{j \in \mathrm{N}_{i_{1}}\left(t_{1}\right)} a_{i_{1} j}\left(t_{1}\right) x_{j}\left(t_{1}\right) \\
& =a_{i_{1} i_{0}}\left(t_{1}\right) x_{i_{0}}\left(t_{1}\right)+\sum_{j \in \mathrm{N}_{i_{1}}\left(t_{1}\right) \backslash\left\{i_{0}\right\}} a_{i_{1} j}\left(t_{1}\right) x_{j}\left(t_{1}\right) \\
& \leq a_{i_{1} i_{0}}\left(t_{1}\right) h\left(C_{0}\right)+\left(1-a_{i_{1} i_{0}}\left(t_{1}\right)\right) H\left(C_{0}\right) \\
& \leq \eta h\left(C_{0}\right)+(1-\eta) H\left(C_{0}\right),
\end{aligned}
$$

which gives

$$
\mathbf{P}\left(x_{i_{1}}\left(t_{1}+1\right) \leq \eta h\left(C_{0}\right)+(1-\eta) H\left(C_{0}\right)\right) \geq P_{t_{1}} .
$$

The fact that the random node updates are independent of the graph process as well as among different nodes, allows us to further conclude that

$$
\mathbf{P}\left(x_{m}\left(t_{1}+1\right) \leq \eta h\left(C_{0}\right)+(1-\eta) H\left(C_{0}\right), m \in\left\{i_{0}\right\} \cup \mathrm{V}_{1}\right) \geq P_{t_{1}}^{\left|\mathrm{V}_{1}\right|} .
$$

Step 2. We continue to define

$$
t_{2}=\inf _{k \geq t_{1}}\left\{\text { there is an arc between } \mathrm{V} \backslash\left(\left\{i_{0}\right\} \cup \mathrm{V}_{1}\right) \text { and }\left\{i_{0}\right\} \cup \mathrm{V}_{1} \text { in } \mathrm{G}_{k}\right\}
$$

and

$$
\mathrm{V}_{2}=\left\{j \in \mathrm{V} \backslash\left(\left\{i_{0}\right\} \cup \mathrm{V}_{1}\right): \text { there is an arc between }\left\{i_{0}\right\} \cup \mathrm{V}_{1} \text { and } j \text { in } \mathrm{G}_{t_{2}}\right\} .
$$

Again $t_{2}$ is a stopping time of the graph process $\left\langle\mathrm{G}_{k}\right\rangle$, and by definition there holds

$$
\mathbf{P}\left(t_{2}<C_{2} \mid t_{1}<C_{1}\right) \geq q
$$

Repeating the similar analysis as Step 1 we obtain

$$
\mathbf{P}\left(x_{m}\left(t_{2}+1\right) \leq \eta^{2} h\left(C_{0}\right)+\left(1-\eta^{2}\right) H\left(C_{0}\right), m \in\left\{i_{0}\right\} \bigcup \mathrm{V}_{1} \bigcup \mathrm{V}_{2}\right) \geq P_{t_{1}}^{\left|\mathrm{V}_{1}\right|} P_{t_{2}}^{\left|\mathrm{V}_{2}\right|}
$$

Step 3. Continuing the analysis, $t_{3}, \ldots, t_{\mu_{0}}$ and $\mathrm{V}_{3}, \ldots \mathrm{V}_{\mu_{0}}$ can be found until

$$
\mathrm{V}=\left\{i_{0}\right\} \bigcup\left(\bigcup_{l=1}^{\mu_{0}} \mathrm{~V}_{l}\right)
$$

for some $\mu_{0} \leq n-1$, and eventually

$$
\mathbf{P}\left(x_{m}\left(t_{\mu_{0}}+1\right) \leq \eta^{\mu_{0}} h\left(C_{0}\right)+\left(1-\eta^{\mu_{0}}\right) H\left(C_{0}\right), m \in \mathrm{V}\right) \geq \prod_{l=1}^{\mu_{0}} P_{t_{l}}^{\left|\mathrm{V}_{l}\right|} .
$$

This gives us

$$
\mathbf{P}\left(\mathcal{H}\left(t_{\mu_{0}}+1\right) \leq\left(1-\eta^{\mu_{0}}\right) \mathcal{H}\left(C_{0}\right)\right) \geq \prod_{l=1}^{\mu_{0}} P_{t_{l}}^{\left|\mathrm{V}_{l}\right|}
$$


Noting that $\mu_{0} \leq n-1$ as well as the fact that $\mathbf{P}\left(t_{\mu_{0}}<C_{n-1}\right) \geq q^{n-1}$ in light of the connectivity independence, we further conclude from (16) that

$$
\mathbf{P}\left(\mathcal{H}\left(C_{n-1}\right) \leq\left(1-\eta^{n-1}\right) \mathcal{H}\left(C_{0}\right)\right) \geq \hat{P}_{0} q^{n-1}
$$

with $\hat{P}_{0}=\inf _{\alpha_{1}<\cdots<\alpha_{n-1}}\left\{\prod_{l=1}^{n-1} P_{\alpha_{l}}: \alpha_{j} \in\left[C_{0}, C_{n-1}\right), j=1, \ldots, n-1\right\}$.

We can recursively apply the above analysis and bounds of $\mathcal{H}\left(C_{s(n-1)}\right)$ can be obtained for $s=1,2, \ldots$ as

$$
\mathbf{P}\left(\mathcal{H}\left(C_{(s+1)(n-1)}\right) \leq\left(1-\eta^{n-1}\right) \mathcal{H}\left(C_{s(n-1)}\right)\right) \geq \hat{P}_{s} q^{n-1}
$$

This ensures global a.s. consensus and that

$$
\mathscr{T}_{\text {com }}(\epsilon) \leq \inf \left\{C_{s(n-1)}: \sum_{i=0}^{s-1} \log \left(1-(q \eta)^{(n-1)} \cdot \hat{P}_{i}\right)^{-1} \geq \log \epsilon^{-2}\right\} .
$$

The proof is now complete.

Remark 2 If we impose the condition that $P_{k+1} \leq P_{k}$ for all $k$ in Proposition 2 , then global a.s. consensus for Algorithm (1) can be guaranteed by $\sum_{s=0}^{\infty} P_{C_{s(n-1)}-1}^{n-1}=\infty$.

\subsection{Acyclic Connections}

A digraph $\mathcal{G}=(\mathrm{V}, \mathcal{E})$ is called acyclic if it contains no directed cycle. This subsection focuses on acyclic graphs.

Let $\mathcal{G}$ be an acyclic, quasi-strongly connected digraph. Then it is not hard to see that $\mathcal{G}$ has one and only one root. Denote this root as $v_{0}$ and let $v_{0} \rightarrow j$ be a path from $v_{0}$ to $j$ in $\mathcal{G}$. Let $\left|v_{0} \rightarrow j\right|$ represent the length of $v_{0} \rightarrow j$ as the number of arcs in this path. We now define a function on the vertices of $\mathcal{G}$ by $\hbar\left(v_{0}\right)=0$ and $\hbar(j)=\max \left\{\left|v_{0} \rightarrow j\right|: v_{0} \rightarrow j\right.$ is a path in $\left.\mathcal{G}\right\}$ for any $j \neq v_{0}$. Let $d_{*}=\max _{i \in \mathrm{V}} \hbar(i)$, which is the maximum path length and therefore is upper bounded by $n-1$. Then we establish the following lemma indicating that this function $\hbar$ is surjective from $\mathrm{V}$ to $\left\{0, \ldots, d_{*}\right\}$.

Lemma 2 Let $\mathcal{G}$ be an acyclic, quasi-strongly connected digraph. Then $\hbar^{-1}(m)=\{i: \hbar(i)=m\}$ is nonempty for all $m=0, \ldots, d_{*}$.

Proof. The conclusion holds for $m=0$ trivially.

Let us prove the conclusion for $m=1$ by contradiction. Assume that $\hbar^{-1}(1)=\emptyset$. Then we have $m_{0} \doteq \inf _{i \neq v_{0}} \hbar(i)>1$. Take a node $j_{0}$ with $\hbar\left(j_{0}\right)=m_{0}$. There exists a (simple) path $v_{0} \rightarrow j_{0}$ in $\mathcal{G}$ with length $m_{0}>1$. Let $v_{*}$ be the node for which $\operatorname{arc}\left(v_{*}, j_{0}\right)$ is included in $v_{0} \rightarrow j_{0}$. According to the definition of $m_{0}$, we have $\hbar\left(v_{*}\right) \geq m_{0}$. Suppose $v_{0} \rightarrow v_{*}$ is a path with length $\hbar\left(v_{*}\right)$. Note that, $j_{0}$ cannot 
be included in $v_{0} \rightarrow v_{*}$ because then it would generate a cycle $j_{0} \rightarrow v_{*} \rightarrow j_{0}$. Consequently, another path $v_{0} \rightarrow v_{*} \rightarrow j_{0}$ is obtained whose length is $\hbar\left(v_{*}\right)+1>m_{0}$. This contradicts the selection rule of $j_{0}$. Therefore, the conclusion holds for $m=1$.

Next, we construct another graph $\mathcal{G}^{*}$ from $\mathcal{G}$ by viewing node set $\left\{v_{0}\right\} \bigcup \hbar^{-1}(1)$ as a single node in the new graph without changing the arcs. We see that $\hbar^{-1}(2)$ of $\mathcal{G}$ is exactly the same as the node set $\hbar^{-1}(1)$ of $\mathcal{G}^{*}$, while the latter is nonempty via previous analysis. Continuing the argument, the conclusion follows.

Here comes our main result for acyclic graphs.

Proposition 3 Let $\mathcal{G}=(\mathrm{V}, \mathcal{E})$ be an acyclic, quasi-strongly connected digraph and assume that a.s. $\mathrm{E}_{k} \subseteq \mathcal{E}$ for all $k$. Suppose $\left\langle\mathrm{G}_{k}\right\rangle$ is infinitely stochastically quasi-strongly connected. Then Algorithm (11) achieves global a.s. consensus if $\sum_{s=0}^{\infty}\left[\inf _{C_{s} \leq \alpha<C_{s+1}} P_{\alpha}\right]=\infty$.

Proof. Let $v_{0}$ be the unique root node of $\mathcal{G}$. Based on Lemma 2, $\mathrm{V}_{i}=\hbar^{-1}(i)$ for $i=0, \ldots, \hbar_{0}$ can be defined satisfying $\mathrm{V}_{0}=\left\{v_{0}\right\}$ and $\mathrm{V}=\bigcup_{i=0}^{d_{*}} \mathrm{~V}_{i}$. Obviously we have $\mathbf{P}\left(x_{v_{0}}(k)=x_{v_{0}}(0), k \geq 0\right)=1$ because with probability one, $v_{0}$ has no neighbor except itself for all $k$. We first investigate the nodes in $\mathrm{V}_{1}$.

Claim. $\mathbf{P}\left(\lim _{k \rightarrow \infty}\left|x_{m}(k)-x_{v_{0}}(0)\right|=0\right)=1$ for all $m \in \mathrm{V}_{1}$.

Take $v_{1} \in \mathrm{V}_{1}$. By our assumption $v_{0}$ is the only neighbor of $v_{1}$ excluding itself in $\mathrm{G}_{k}$ for all $k$. Define $t_{1}=\inf _{k \geq 0}\left\{\left(v_{0}, v_{1}\right) \in \mathrm{E}_{k}\right\}$. Then $\mathbf{P}\left(t_{1}<C_{1}\right) \geq q$. We have

$$
\begin{aligned}
\left|\sum_{j \in \mathrm{N}_{v_{1}}\left(t_{1}\right)} a_{v_{1} j}\left(t_{1}\right) x_{j}\left(t_{1}\right)-x_{v_{0}}(0)\right| & =\left|a_{v_{1} v_{0}}\left(t_{1}\right) x_{v_{0}}\left(t_{1}\right)+a_{v_{1} v_{1}}\left(t_{1}\right) x_{v_{1}}\left(t_{1}\right)-x_{v_{0}}(0)\right| \\
& =\left(1-a_{v_{1} v_{0}}\left(t_{1}\right)\right)\left|x_{v_{1}}(0)-x_{v_{0}}(0)\right| \\
& \leq(1-\eta)\left|x_{v_{1}}(0)-x_{v_{0}}(0)\right|,
\end{aligned}
$$

which yields

$$
\mathbf{P}\left(\left|x_{v_{1}}\left(t_{1}+1\right)-x_{v_{0}}(0)\right| \leq(1-\eta)\left|x_{v_{1}}(0)-x_{v_{0}}(0)\right|\right) \geq P_{t_{1}}
$$

Thus, we obtain

$$
\mathbf{P}\left(\left|x_{v_{1}}\left(C_{1}\right)-x_{v_{0}}(0)\right| \leq(1-\eta)\left|x_{v_{1}}(0)-x_{v_{0}}(0)\right|\right) \geq \tilde{P}_{0} q
$$

where $\tilde{P}_{0}=\inf _{C_{0} \leq \alpha<C_{1}} P_{\alpha}$. Repeating the analysis on time interval $\left[C_{m}, C_{m+1}\right), m=1,2, \ldots$, we have

$$
\mathbf{P}\left(\left|x_{v_{1}}\left(C_{m+1}\right)-x_{v_{0}}(0)\right| \leq(1-\eta)\left|x_{v_{1}}\left(C_{m}\right)-x_{v_{0}}(0)\right|\right) \geq \tilde{P}_{m} q, \quad m=1,2, \ldots
$$

Then connectivity independence leads to $\mathbf{P}\left(\lim _{k \rightarrow \infty}\left|x_{v_{1}}(k)-x_{v_{0}}(0)\right|=0\right)=1$. The claim is proved.

Next, we turn to $\mathrm{V}_{2}$. The analysis of nodes in $\mathrm{V}_{2}$ will be carried out via a sample-path argument. Let $\ell \geq 1$. The above claim we just proved implies that there exists a random variable $T_{\ell}(\omega)>0$ which is a.s. 
finite, such that

$$
\mathbf{P}\left(\left|x_{m}(k)-x_{v_{0}}(0)\right| \leq 1 / \ell, k \geq T_{\ell}, m \in \mathrm{V}_{1}\right)=1 .
$$

Take $v_{2}$ as an arbitrary node in $\mathrm{V}_{2}$. We prove $\mathbf{P}\left(\lim \sup _{k \rightarrow \infty}\left|x_{v_{2}}(k)-x_{v_{0}}(0)\right| \leq 1 / \ell\right)=1$. Note that from the definition of $\mathrm{V}_{2}$, the state of node $v_{2}$ can only be influenced by nodes in $\left\{v_{0}\right\} \bigcup \mathrm{V}_{1}$ in the entire time horizon.

Define event $\mathscr{E}=\left\{\left|x_{v_{2}}(k)-x_{v_{0}}(0)\right|>1 / \ell, k \geq T_{\ell}\right\}$. There will be two cases.

(i) Since $v_{2}$ can only possibly connect to nodes in $\left\{v_{0}\right\} \cup \mathrm{V}_{1}$, there must hold

$$
\left|x_{v_{2}}(s)-x_{v_{0}}(0)\right| \leq 1 / \ell, s \geq k
$$

if $\left|x_{v_{2}}(k)-x_{v_{0}}(0)\right| \leq 1 / \ell$ for $k \geq T_{\ell}$. This is equivalent to that

$$
\mathbf{P}\left(\limsup _{k \rightarrow \infty}\left|x_{v_{2}}(k)-x_{v_{0}}(0)\right| \leq 1 / \ell \mid \mathscr{E}^{c}\right)=1 .
$$

(ii) By our assumption, $\left\langle\mathrm{G}_{k}\right\rangle$ is infinitely stochastically quasi-strongly connected, and $\sum_{s=0}^{\infty} \tilde{P}_{s}=\infty$ with $\tilde{P}_{s}=\inf _{C_{s} \leq \alpha<C_{s+1}} P_{\alpha}$. Moreover, whenever $\mathrm{G}\left(\left[C_{m}, C_{m+1}\right)\right)$ is quasi-strongly connected, there must be a time instant between $\left[C_{m}, C_{m+1}\right)$ at which there is an arc from $\left\{v_{0}\right\} \cup \mathrm{V}_{1}$ to $v_{2}$. Therefore, applying the Borel-Cantelli lemma we see that a.s. there is an infinite sequence of (random) time instants

$$
t_{1}<t_{2}<\cdots<t_{m}<\cdots
$$

where at each time $t_{m}$, there is at least one arc from $\left\{v_{0}\right\} \cup V_{1}$ to $v_{2}$ and $v_{2}$ successfully updates its state. Then we obtain for all $t_{m} \geq T_{\ell}$ that

$$
\begin{aligned}
& \left|x_{v_{2}}\left(t_{m}+1\right)-x_{v_{0}}(0)\right| \\
& =\left|\sum_{j \in \mathrm{N}_{v_{2}}\left(t_{m}\right)} a_{v_{2} j}\left(t_{m}\right) x_{j}\left(t_{m}\right)-x_{v_{0}}(0)\right| \\
& =\sum_{j \in \mathrm{N}_{v_{2}}\left(t_{m}\right): j \in\left\{v_{0}\right\} \cup \mathrm{V}_{1}} a_{v_{2} j}\left(t_{m}\right)\left|x_{j}\left(t_{m}\right)-x_{v_{0}}(0)\right|+a_{v_{2} v_{2}}\left(t_{m}\right)\left|x_{v_{2}}\left(t_{m}\right)-x_{v_{0}}(0)\right| \\
& \leq\left[1-a_{v_{2} v_{2}}\left(t_{m}\right)\right] \frac{1}{\ell}+a_{v_{2} v_{2}}\left(t_{m}\right)\left|x_{v_{2}}\left(t_{m}\right)-x_{v_{0}}(0)\right| \\
& \leq[1-\eta] \frac{1}{\ell}+\eta\left|x_{v_{2}}\left(t_{m}\right)-x_{v_{0}}(0)\right|
\end{aligned}
$$

which reads

$$
\left|x_{v_{2}}\left(t_{m}+1\right)-x_{v_{0}}(0)\right|-\frac{1}{\ell} \leq \eta\left(\left|x_{v_{2}}\left(t_{m}\right)-x_{v_{0}}(0)\right|-\frac{1}{\ell}\right)
$$

for all $t_{m} \geq T_{\ell}$. Noticing the definition of $t_{m}$, this has already proved that

$$
\mathbf{P}\left(\limsup _{k \rightarrow \infty}\left|x_{v_{2}}(k)-x_{v_{0}}(0)\right| \leq 1 / \ell \mid \mathscr{E}\right)=1 .
$$


As a result, we can now conclude that

$$
\mathbf{P}\left(\limsup _{k \rightarrow \infty}\left|x_{v_{2}}(k)-x_{v_{0}}(0)\right| \leq 1 / \ell\right)=1
$$

Now that $\ell$ is chosen arbitrarily and $v_{2}$ is an arbitrary node in $\mathrm{V}_{2}$, we further obtain

$$
\mathbf{P}\left(\lim _{k \rightarrow \infty}\left|x_{m}(k)-x_{v_{0}}(0)\right|=0\right)=1, \quad m \in \mathrm{V}_{2} .
$$

Finally, a recursive analysis for node sets $\mathrm{V}_{3}, \ldots, \mathrm{V}_{d_{*}}$ eventually gives $\mathbf{P}\left(\lim _{k \rightarrow \infty}\left|x_{m}(k)-x_{v_{0}}(0)\right|=\right.$ $0)=1$ for all $m \in \mathrm{V}$. The desired conclusion thus follows.

Remark 3 Proposition 3 immediately implies the following interesting observations when there holds $\mathrm{E}_{k} \subseteq \mathcal{E}$ for all $k$ for some acyclic, quasi-strongly connected digraph $\mathcal{G}=(\mathrm{V}, \mathcal{E})$.

(i). If $\left\langle\mathrm{G}_{k}\right\rangle$ is infinitely stochastically quasi-strongly connected, and $P_{k+1} \leq P_{k}$ for all $k$, then Algorithm (11) achieves global a.s. consensus when $\sum_{m=0}^{\infty} P_{C_{m}}=\infty$.

(ii). Suppose $\left\langle\mathrm{G}_{k}\right\rangle$ is uniformly stochastically quasi-strongly connected with respect to $B \geq 1$. Let either $B=1$ or $P_{k+1} \leq P_{k}, k \geq 0$. Then Algorithm (1) achieves global a.s. consensus if and only if $\sum_{k=0}^{\infty} P_{k}=\infty$.

\section{Arc-Independent Graphs}

In this section, we investigate the convergence with an arc-independent random graph process and prove Theorem 3. We present the convergence analysis using a stochastic matrix argument.

Let $e_{i}=(0 \ldots 1 \ldots 0)^{\mathrm{T}}$ be the $n \times 1$ unit vector with the $i$ th component equal to one. Denote $\bar{a}_{i}(k)=$ $\left(\bar{a}_{i 1}(k) \ldots \bar{a}_{i n}(k)\right)^{\mathrm{T}}$ as an $n \times 1$ vector with $\bar{a}_{i j}(k)=a_{i j}(k)$ if $j \in \mathrm{N}_{i}(k)$, and $\bar{a}_{i j}(k)=0$ otherwise. Let $\chi_{i}(k), i=1, \ldots, n, k \in \mathbb{N}$ be a sequence of independent Bernoulli random variables satisfying $\mathbf{E}\left[\chi_{i}(k)\right]=$ $P_{k}$. Denote $W(k)=\left(w_{1}(k) \ldots w_{n}(k)\right)^{\mathrm{T}} \in \mathbb{R}^{n \times n}$ as a random matrix with

$$
w_{i}(k)=\chi_{i}(k) \bar{a}_{i}(k)+\left(1-\chi_{i}(k)\right) e_{i}, i=1, \ldots, n .
$$

Algorithm (11) can be written into a compact form:

$$
x(k+1)=W(k) x(k) .
$$

In the remainder of this section, we first establish several useful lemmas on the product of stochastic matrices, and then the proof of Theorem 3 is presented. 


\subsection{Stochastic Matrices}

A finite square matrix $M=\left[M_{i j}\right] \in \mathbb{R}^{n \times n}$ is called stochastic if $M_{i j} \geq 0$ for all $i, j$ and $\sum_{j=1}^{n} M_{i j}=1$ for all $i[32]$. For a stochastic matrix $M$, introduce

$$
\delta(M)=\max _{j} \max _{\alpha, \beta}\left|M_{\alpha j}-M_{\beta j}\right|, \quad \lambda(M)=1-\min _{\alpha, \beta} \sum_{j} \min \left\{M_{\alpha j}, M_{\beta j}\right\} .
$$

The $\lambda(M)$ is often called the Dobrushin's ergodicity coefficient [50]. Note that $\delta(M)$ describes how much the weights assigned by two nodes $\alpha, \beta$ to a node $j$ can differ, and $\lambda(M)=0$ if and only if for each pair of nodes $\alpha$ and $\beta$, for each node $j$ either $\alpha$ or $\beta$ has no weight to $j$. If $\lambda(M)<1, M$ a called a scrambling matrix. A scrambling matrix does not have two orthogonal rows, which means that any two rows share at least one column, where both of them have strictly positive elements. The following lemma can be found in $[16]$.

Lemma 3 For any $k(k \geq 1)$ stochastic matrices $M_{1}, \ldots, M_{k}$, there holds

$$
\delta\left(M_{1} M_{2} \ldots M_{k}\right) \leq \prod_{i=1}^{k} \lambda\left(M_{i}\right) .
$$

Let $M=\left[M_{i j}\right] \in \mathbb{R}^{n \times n}$ be a matrix with nonnegative entries. We can associate a unique digraph $\mathcal{G}_{M}=\left(\mathrm{V}, \mathcal{E}_{M}\right)$ with $M$ on node set $\mathrm{V}=\{1, \ldots, n\}$ such that $(j, i) \in \mathcal{E}_{M}$ if and only if $M_{i j}>0$. We call $\mathcal{G}_{M}$ the induced graph of $M$.

The following lemma is given on the induced graphs of products of stochastic matrices.

Lemma 4 Let $M_{1}, \ldots, M_{k}$ be $k \geq 1$ stochastic matrices with positive diagonal entries. Then $\left(\bigcup_{i=1}^{k} \mathrm{G}_{M_{i}}\right) \subseteq$ $\mathrm{G}_{M_{k} \cdots M_{1}}$.

Proof. We prove the case $k=2$, and the conclusion will then follow by induction.

Denote $\left[M_{1}\right]_{i j},\left[M_{2}\right]_{i j}$ and $\left[M_{2} M_{1}\right]_{i j}$ as the $i j$-entries of $M_{1}, M_{2}$ and $M_{2} M_{1}$, respectively. Note that, we have

$$
\left[M_{2} M_{1}\right]_{i j}=\sum_{m=1}^{n}\left[M_{2}\right]_{i m}\left[M_{1}\right]_{m j} \geq\left[M_{2}\right]_{i i}\left[M_{1}\right]_{i j}+\left[M_{2}\right]_{i j}\left[M_{1}\right]_{j j}
$$

The desired conclusion follows immediately from the fact that $\left[M_{2}\right]_{i i},\left[M_{1}\right]_{j j}>0$.

The following lemma helps in determining whether a product of stochastic matrices is a scrambling matrix.

Lemma 5 Let $M_{1}, \ldots, M_{n-1}$ be $n-1$ stochastic matrices with positive diagonal entries. Suppose there exists a node $i_{0} \in \mathrm{V}$ such that $i_{0}$ is a root of $\mathcal{G}_{M_{\tau}}$ for all $\tau=1, \ldots, n-1$. Then $M_{n-1} \cdots M_{1}$ is a scrambling matrix. 
Proof. Since $i_{0}$ is a root of $\mathcal{G}_{M_{1}}$, at least one node $i_{1}$ exists different from $i_{0}$ such that $\left(i_{0}, i_{1}\right) \in \mathcal{E}_{M_{1}}$. This immediately implies $\left[M_{1}\right]_{i_{1} i_{0}}>0$ according to the definition of induced graph.

Further, according to Lemma 4, we have $\left[M_{2} M_{1}\right]_{i_{1} i_{0}}>0$ resulting from $\left[M_{1}\right]_{i_{1} i_{0}}>0$. Since $i_{0}$ is also a root of $\mathcal{G}_{M_{2}}$, there must be a node $i_{2}$ different with $i_{0}$ and $i_{1}$ such that there is at least one arc leaving from $\left\{i_{0}, i_{1}\right\}$ entering $i_{2}$ in $\mathcal{G}_{M_{2}}$. There will be two cases.

(i) When $\left(i_{0}, i_{2}\right) \in \mathcal{E}_{M_{2}}$, there holds $\left[M_{2}\right]_{i_{2} i_{0}}>0$. This implies $\left[M_{2} M_{1}\right]_{i_{2} i_{0}}>0$ based on Lemma 4 .

(ii) When $\left(i_{1}, i_{2}\right) \in \mathcal{E}_{M_{2}}$, there holds $\left[M_{2}\right]_{i_{2} i_{1}}>0$. In this case we have

$$
\left[M_{2} M_{1}\right]_{i_{2} i_{0}}=\sum_{\tau=1}^{n}\left[M_{2}\right]_{i_{2} \tau}\left[M_{1}\right]_{\tau i_{0}} \geq\left[M_{2}\right]_{i_{2} i_{1}}\left[M_{1}\right]_{i_{1} i_{0}}>0 .
$$

We recursively carry out the above analysis, and then $i_{3}, \ldots, i_{n-1}$ can be found with $\mathrm{V}=\left\{i_{0}, \ldots, i_{n-1}\right\}$ such that

$$
\left[M_{n-1} \cdots M_{1}\right]_{i_{m} i_{0}}>0
$$

for all $m=0,1, \ldots, n-1$. According to the definition of $\delta(\cdot)$, (21) implies

$$
\lambda\left(M_{n-1} \cdots M_{1}\right) \leq 1-\min _{m=0, \ldots, n-1}\left[M_{n-1} \cdots M_{1}\right]_{i_{m} i_{0}}<1 .
$$

The desired lemma follows.

\subsection{Proof of Theorem [3: Convergence}

This subsection presents the proof of the conclusion on a.s. consensus in Theorem 3, We only need to show the sufficiency part. Note that global a.s. consensus of (11) is equivalent to

$$
\mathbf{P}\left(\lim _{k \rightarrow \infty} \delta(W(k) \cdots W(0))=0\right)=1 .
$$

We define $\Psi_{k}=\mathrm{I}_{\sum_{i=1}^{n} \chi_{k}}$ with I representing the indicator function. In other words,

$$
\Psi_{k}= \begin{cases}1, & \text { if at least one } \chi_{i}(k)=1 \text { at } k \\ 0, & \text { otherwise }\end{cases}
$$

Then we have $\Psi_{k}=1$ with probability $1-\left(1-P_{k}\right)^{n}$ and $\Psi_{k}=0$ with probability $\left(1-P_{k}\right)^{n}$. Moreover, $\Psi_{0}, \Psi_{1}, \ldots$ are independent. Applying the Borel-Cantelli Lemma to $\left\langle\chi_{i}(k)\right\rangle, i=1, \ldots, n$ and invoking the independence $\Psi_{k}$ we conclude the following property of $\Psi_{k}$.

Lemma $6 \mathbf{P}\left(\Psi_{k}=1\right.$ for infinitely many $\left.k\right)=1$ if and only if $\sum_{k=0}^{\infty} P_{k}=\infty$. 
Noting the fact that

$$
1-n y \leq(1-y)^{n}
$$

for all $y \in[0,1]$ and $n \geq 1$, there holds for all $k$ that

$$
1-\left(1-P_{k}\right)^{n} \leq n P_{k}
$$

As a result, we obtain

$$
\mathbf{P}\left(\chi_{i}(k)=1 \mid \Psi_{k}=1\right)=\frac{P_{k}}{1-\left(1-P_{k}\right)^{n}} \geq \frac{P_{k}}{n P_{k}}=\frac{1}{n}
$$

for all $i$ and $k$.

Next, we introduce the following sequence (known as the Bernoulli sequence of $\left\langle\Psi_{k}\right\rangle$ ):

$$
\zeta_{1}<\ldots<\zeta_{m}<\zeta_{m+1}<\ldots
$$

where $\zeta_{m}$ is the $m^{\prime}$ th time when $\Psi_{k}=1$. The $\zeta_{m}$ are stopping times of the sequence $\left\langle\Psi_{k}\right\rangle$, and Lemma [5 ensures that each $\zeta_{m}$ is almost surely finite when $\sum_{k=0}^{\infty} P_{k}=\infty$. For any $(i, j) \in \mathcal{E}^{\dagger}$, there hold for all $m=1,2, \ldots$ that

$$
\begin{aligned}
& \mathbf{P}\left((i, j) \in \mathrm{G}_{W\left(\zeta_{m}\right)}\right) \\
& \stackrel{a)}{=} \mathbf{P}\left(\chi_{j}\left(\zeta_{m}\right)=1 \text { and }(i, j) \in \mathrm{G}_{\zeta_{m}}\right) \\
& \stackrel{b)}{=} \mathbf{P}\left(\chi_{j}\left(\zeta_{m}\right)=1\right) \cdot \mathbf{P}\left((i, j) \in \mathrm{G}_{\zeta_{m}}\right) \\
& \stackrel{c)}{\geq} \frac{q_{*}}{n},
\end{aligned}
$$

where $a$ ) is from the structure of the algorithm, $b$ ) is from the independence between the random graph process and the $\chi_{i}(k)$, and $c$ ) holds from (22).

Denote $Q_{1}=W\left(\zeta_{\left|\mathcal{E}^{\dagger}\right|}\right) \cdots W\left(\zeta_{2}\right) W\left(\zeta_{1}\right)$, where $\left|\mathcal{E}^{\dagger}\right|$ represents the number of elements in $\mathcal{E}^{\dagger}$. From (24), we can pick up $\left(i_{\tau}, j_{\tau}\right), \tau=1, \ldots,\left|\mathcal{E}^{\dagger}\right|$ as all the $\operatorname{arcs}$ in $\mathcal{E}^{\dagger}$, and the arc-independence (cf., the $\zeta_{m}$ are stopping times of i.i.d. random process $\left\langle\Psi_{k}\right\rangle$, and the random graph process $\left\langle\mathrm{G}_{k}\right\rangle$ is independent with the $\Psi_{k}$ ) leads to

$$
\mathbf{P}\left(\left(i_{\tau}, j_{\tau}\right) \in \mathrm{G}_{W\left(\zeta_{\tau}\right)}, \tau=1, \ldots,\left|\mathcal{E}^{\dagger}\right|\right) \geq\left(\frac{q_{*}}{n}\right)^{\left|\mathcal{E}^{\dagger}\right|}
$$

which yields

$$
\mathbf{P}\left(\mathcal{G}^{\dagger} \subseteq \mathrm{G}_{Q_{1}}\right) \geq \mathbf{P}\left(\mathcal{G}^{\dagger} \subseteq\left(\bigcup_{\tau=1}^{\left|\mathcal{E}^{\dagger}\right|} \mathrm{G}_{W\left(\zeta_{\tau}\right)}\right)\right) \geq\left(\frac{q_{*}}{n}\right)^{\left|\mathcal{E}^{\dagger}\right|}
$$

according to Lemma 4 . 
We continue to define $Q_{m}=W\left(\zeta_{m\left|\mathcal{E}^{\dagger}\right|}\right) \cdots W\left(\zeta_{(m-1)\left|\mathcal{E}^{\dagger}\right|+1}\right)$ for $m=2,3, \ldots$, and similarly

$$
\mathbf{P}\left(\mathcal{G}^{\dagger} \subseteq \mathrm{G}_{Q_{m}}\right) \geq\left(\frac{q_{*}}{n}\right)^{\left|\mathcal{E}^{\dagger}\right|}
$$

for all $m$. Because $\mathcal{G}^{\dagger}$ is quasi-strongly connected, Lemma 5 yields

$$
\mathbf{P}\left(\lambda\left(Q_{n-1} \cdots Q_{1}\right)<1\right) \geq\left(\frac{q_{*}}{n}\right)^{(n-1)\left|\mathcal{E}^{\dagger}\right|} .
$$

Moreover, $Q_{n-1} \cdots Q_{1}$ represents a product of $(n-1)\left|\mathcal{E}^{\dagger}\right|$ stochastic matrices, each of which satisfies the weights rule. Therefore, there holds

$$
\left[Q_{n-1} \cdots Q_{1}\right]_{i j} \geq \eta^{(n-1)\left|\mathcal{E}^{\dagger}\right|}
$$

for every nonzero entry $\left[Q_{n-1} \cdots Q_{1}\right]_{i j}$ of $Q_{n-1} \cdots Q_{1}$.

We see from (25) and (26) that

$$
\mathbf{P}\left(\lambda\left(Q_{n-1} \cdots Q_{1}\right) \leq 1-\eta^{(n-1)\left|\mathcal{E}^{\dagger}\right|}\right) \geq\left(\frac{q_{*}}{n}\right)^{(n-1)\left|\mathcal{E}^{\dagger}\right|} .
$$

Denoting $U_{m}=Q_{m(n-1)} \cdots Q_{(m-1)(n-1)+1}$ for $m=1,2, \ldots$, we can now further conclude for all $m=$ $1,2, \ldots$ that

$$
\mathbf{P}\left(\lambda\left(U_{m}\right) \leq 1-\eta^{(n-1)\left|\mathcal{E}^{\dagger}\right|}\right) \geq\left(\frac{q_{*}}{n}\right)^{(n-1)\left|\mathcal{E}^{\dagger}\right|}
$$

Thus, based on Lemma 3, we have

$$
\lim _{m \rightarrow \infty} \mathbf{E}\left[\delta\left(U_{m} \cdots U_{1}\right)\right] \leq \lim _{m \rightarrow \infty} \mathbf{E}\left[\prod_{\tau=1}^{m} \lambda\left(U_{m}\right)\right]=0,
$$

Then from Fatou's Lemma we have

$$
\mathbf{P}\left(\lim _{m \rightarrow \infty} \delta\left(U_{m} \cdots U_{1}\right)=0\right)=1
$$

which leads to

$$
\mathbf{P}\left(\lim _{k \rightarrow \infty} \delta(W(k) \cdots W(0))=0\right)=1
$$

since $W(k)=I_{n}$ for any $k \notin\left\{\zeta_{1}, \zeta_{2}, \ldots\right\}$ ( $I_{n}$ is the identity matrix). Hence, we have proved global a.s. consensus for Algorithm (1).

\subsection{Proof of Theorem 3: Computation Time}

In this subsection, we establish the upper bound of $\mathscr{T}_{\text {com }}(\epsilon)$ given in Theorem 3 , 
Denote $\Theta_{k}=W(k-1) \ldots W(0)$. For all $i, j$ and $\tau$, there holds

$$
\begin{aligned}
\left|x_{i}(k)-x_{j}(k)\right| & =\left|\sum_{\alpha=1}^{n}\left[\Theta_{k}\right]_{i \alpha} x_{\alpha}(0)-\sum_{\alpha=1}^{n}\left[\Theta_{k}\right]_{j \alpha} x_{\alpha}(0)\right| \\
& =\left|\sum_{\alpha=1}^{n}\left[\Theta_{k}\right]_{i \alpha}\left(x_{\alpha}(0)-x_{\tau}(0)\right)-\sum_{\alpha=1}^{n}\left[\Theta_{k}\right]_{j \alpha}\left(x_{\alpha}(0)-x_{\tau}(0)\right)\right| \\
& \leq \sum_{\alpha=1}^{n}\left|\left[\Theta_{k}\right]_{i \alpha}-\left[\Theta_{k}\right]_{j \alpha}\right| \cdot \max _{\alpha}\left|x_{\alpha}(0)-x_{\tau}(0)\right| \\
& \leq n \delta\left(\Theta_{k}\right) \cdot \max _{\alpha}\left|x_{\alpha}(0)-x_{\tau}(0)\right| .
\end{aligned}
$$

Therefore, we obtain that for all $k \geq 1$,

$$
\mathcal{H}(k) \leq n \delta\left(\Theta_{k}\right) \mathcal{H}(0)
$$

Introduce

$$
\xi_{k}:=\sum_{i=0}^{k-1} \Psi_{i}
$$

for $k=0,1, \ldots$ Then there holds

$$
\xi_{k}=\max \left\{m: \zeta_{m} \leq k-1\right\}
$$

Denote $E_{0}=(n-1)\left|\mathcal{E}^{\dagger}\right|$. Then according to Lemma 3 and applying Markov's Inequality, (28) implies

$$
\begin{aligned}
\mathbf{P}\left(\frac{\mathcal{H}(k)}{\mathcal{H}(0)} \geq \epsilon\right) & \leq \mathbf{P}\left(\delta\left(\Theta_{k}\right) \geq \frac{\epsilon}{n}\right) \\
& \leq \frac{n}{\epsilon} \mathbf{E}\left[\delta\left(\Theta_{k}\right)\right] \\
& \leq \frac{n}{\epsilon} \mathbf{E}\left[\lambda_{\left\lfloor\frac{\xi_{k}}{E_{0}}\right\rfloor} \cdots \lambda_{1}\right],
\end{aligned}
$$

where $E_{0}=(n-1)\left|\mathcal{E}^{\dagger}\right|$, and by definition $\lambda_{m}=\lambda\left(U_{m}\right)$ with $U_{m}$ introduced in the previous subsection. Here $\lfloor z\rfloor$ represents the largest integer no greater than $z$.

From (25) we conclude that

$$
\mathbf{E}\left[\lambda_{m}\right] \leq 1-\left(\frac{\eta q_{*}}{n}\right)^{E_{0}}
$$

for all $m$. This further yields

$$
\begin{aligned}
\mathbf{E}\left[\lambda_{\left\lfloor\frac{\xi_{k}}{E_{0}}\right\rfloor} \ldots \lambda_{1}\right] & =\mathbf{E}\left[\mathbf{E}\left[\lambda_{\left\lfloor\frac{\xi_{k}}{E_{0}}\right\rfloor} \ldots \lambda_{1} \mid \xi_{k}\right]\right] \\
& \leq\left(1-\left(\frac{\eta q_{*}}{n}\right)^{E_{0}}\right)^{\mathbf{E}\left[\left\lfloor\frac{\xi_{k}}{E_{0}}\right]\right]} \\
& \leq\left(1-\left(\frac{\eta q_{*}}{n}\right)^{E_{0}}\right)^{\sum_{i=0}^{k-1} \mathbf{E}\left[\Psi_{i}\right] / E_{0}-1} \\
& =\left(1-\left(\frac{\eta q_{*}}{n}\right)^{E_{0}}\right)^{\sum_{i=0}^{k-1}\left[1-\left(1-P_{i}\right)^{n}\right] / E_{0}-1}
\end{aligned}
$$


Combining (29) and (30), we obtain

$$
\mathbf{P}\left(\frac{\mathcal{H}(k)}{\mathcal{H}(0)} \geq \epsilon\right) \leq \frac{n}{\epsilon}\left(1-\left(\frac{\eta q_{*}}{n}\right)^{E_{0}}\right)^{\sum_{i=0}^{k-1}\left[1-\left(1-P_{i}\right)^{n}\right] / E_{0}-1}
$$

based on which the desired upper bound of $\mathscr{T}_{\text {com }}(\epsilon)$ follows immediately from some simple algebra.

\section{Examples, Mixing, and Gossiping}

In this section, we first investigate a few basic random graph models from the literature discussing the applicability of the previously established results. Next, we extend the concepts of connectivity-independence and arc-independence to *-mixing of random graphs, and establish the corresponding a.s. consensus convergence result. An immediate advantage of this extension is that the majority of random graph models is covered by $*$-mixing random graphs. Finally, we look further into random gossiping algorithms and present results on a.s. finite-time consensus for this important class of random algorithms.

\subsection{Examples}

We discuss a few random graph examples and show the applicability of the connectivity and arc independence. Apparently all independent random graph processes are immediately connectivity independent, but not necessarily arc independent.

Let $\mathrm{K}_{n}$ denote the complete digraph with $n$ nodes, i.e., $(i, j) \in \mathrm{K}_{n}$ for all $i \neq j \in \mathrm{V}$.

Example 1 [Erdős-Rényi]. The random graph process $\left\langle\mathrm{G}_{k}\right\rangle$ is a sequence of i.i.d. bidirectional or directed Erdős-Rényi random graphs. A bidirectional Erdős-Rényi graph is a random graph over node set V such that independently with a probability $p$, there is a bidirectional edge between every pair of nodes in V. A directed Erdős-Rényi graph is defined in that independently with a probability $p$, there is a directed arc $(i, j)$ for every ordered pair $(i, j)$ of nodes in V. Consensus dynamics over Erdős-Rényi random graphs were studied in [21, 22].

Both the directed and bidirectional Erdős-Rényi graph processes are arc-independent with respect to the basic graph $\mathrm{K}_{n}$. Note that arc appearance in a fixed bidirectional Erdős-Rényi random graph $G_{k}$ is not independent, since $(i, j) \in \mathrm{E}_{k}$ implies $(j, i) \in \mathrm{E}_{k}$.

Example 2 [Random Link Failure]. Let $\mathcal{G}^{\mathrm{u}}=\left(\mathrm{V}, \mathcal{E}^{\mathrm{u}}\right)$ be an underlying digraph representing fundamentally who can possibly talk to whom in the network. Independently at different time instants and among different $\operatorname{arcs}$ in $\mathcal{E}^{\mathrm{u}},(i, j) \in \mathrm{E}_{k}$ with a given probability $p_{i j}>0$. In this way we obtain an i.i.d. random graph process $\left\langle\mathrm{G}_{k}\right\rangle$. This model describes independent random failure in node communications, and consensus algorithm with random link failure is studied in [27, 26, 28, 24]. Then we see $\left\langle\mathrm{G}_{k}\right\rangle$ given by this random link failure process is arc-independent with respect to the basic graph $\mathcal{G}^{\mathrm{u}}$. 
Example 3 [Markovian Random Graph]. The random graph process $\left\langle\mathrm{G}_{k}\right\rangle$ is generated by a Markov chain whose state space is (or, a subset of) $\mathscr{G}$. Such examples were studied in [19, 29]. Since Markovian random graphs introduce local dependence in time, in general it will be difficult for $\left\langle\mathrm{G}_{k}\right\rangle$ to be either connectivity or arc independent. However, if the Markov chain is defined on the subset of quasi-strongly connected digraphs in $\mathscr{G}$, then connectivity-independence holds trivially for $\left\langle\mathrm{G}_{k}\right\rangle$.

Example 4 [Random Gossiping]. Independently at each time step, a pair of nodes are randomly selected for averaging their states, called gossiping. Comprehensive analysis for random gossiping algorithms is established in [2], and gossiping algorithm also serves as a model for social belief propagation [10, 11]. An independent random gossiping process $\left\langle\mathrm{G}_{k}\right\rangle$ is arc-independent and trivially connectivity-independent (the probability of $\mathrm{G}_{k}$ being quasi-strongly connected equals zero if $n>2$, and one if $n=2$ ). We can consider the random joint graphs $\mathrm{G}([m T,(m+1) T-1])$ over time intervals $[m T,(m+1) T-1]$ for $m \in \mathbb{N}$, in which $\langle\mathrm{G}([m T,(m+1) T-1])\rangle$ is both connectivity and arc independent, and for sufficiently large integer $T$ the condition of Proposition 1 will be satisfied.

\subsection{Connectivity-Mixing and Arc-Mixing Random Graphs}

In the previous subsection, we have illustrated that the concepts of connectivity/arc independence can be applied to many independent random graph models, but become restrictive with non-independent, e.g., Markovian random graph processes. We now propose an alternative method from mixing theory, which, inherits the spirit of a network version of Borel-Cantelli lemma in the presence of connectivity/arc independence (and many our analysis techniques smoothly apply), however allows for possible weakening of the independence assumption.

\subsubsection{Arc/Connectivity $*$-Mixing}

First we recall some basic concepts and results from mixing of random processes (cf., 47] for a comprehensive survey). Let $\left\langle X_{k}\right\rangle$ be a sequence of random variables, where each $X_{k}$ is taken from a common probability space $(\Omega, \mathcal{F}, \mathbb{P})$. For the ease of presentation we continue to use $(\Omega, \mathcal{F}, \mathbb{P})$ to denote the product probability space where $\left(X_{k}, k \in \mathbb{N}\right)$ lies in, the existence of which is guaranteed by the Kolmogorov's extension theorem [35]. The $\sigma$-algebra generated by $X_{a}, \cdots, X_{b}$ is denoted as $\mathcal{F}_{a}^{b}$ for $0 \leq a \leq b \leq \infty$. The tail $\sigma$-algebra of $\left\langle X_{k}\right\rangle$, denoted $\mathcal{T}$, is defined by $\mathcal{T}=\bigcap_{m=0}^{\infty} \mathcal{F}_{m}^{\infty}$. Events belonging to $\mathcal{T}$ are called tail events. For a sequence of events $\left\langle\mathcal{A}_{k}\right\rangle$ with $\mathcal{A}_{k} \in \mathcal{F}$, we define $\mathrm{I}_{\mathcal{A}_{k}}$ as their indicators, i.e., $\mathrm{I}_{\mathcal{A}_{k}}(\omega)=1$ if $\omega \in \mathcal{A}_{k}$ and $\mathrm{I}_{\mathcal{A}_{k}}(\omega)=0$ otherwise for all $k \in \mathbb{N}$. Then $\left\langle\mathrm{I}_{\mathcal{A}_{k}}\right\rangle$ is a sequence of random variables over 
$(\Omega, \mathcal{F}, \mathbb{P})$. We quote the following definition from [46] 4 .

Definition 5 A sequence of random variables $\left\langle X_{k}\right\rangle$ is called $*$-mixing if there exists a non-increasing sequence of real numbers $\left\langle f_{m}\right\rangle$ with $\lim _{m \rightarrow \infty} f_{m}=0$, such that there holds

$$
|\mathbb{P}(\mathcal{A} \cap \mathcal{B})-\mathbb{P}(\mathcal{A}) \mathbb{P}(\mathcal{B})| \leq f_{m} \mathbb{P}(\mathcal{A}) \mathbb{P}(\mathcal{B}), \mathcal{A} \in \mathcal{F}_{0}^{t}, \mathcal{B} \in \mathcal{F}_{t+m}^{\infty}
$$

for all $t \in \mathbb{N}$. A sequence of events $\left\langle\mathcal{A}_{k}\right\rangle$ is $*$-mixing if $\left\langle\mathrm{I}_{\mathcal{A}_{k}}\right\rangle$ is $*$-mixing.

Associated with $\left\langle\mathrm{G}_{k}\right\rangle$, we let $\left\langle\mathcal{C}_{k}\right\rangle$ be the sequence of connectivity events defined by

$$
\mathcal{C}_{k}:=\left\{\omega: \mathrm{G}_{k}(\omega) \text { is quasi-strongly connected }\right\}, \quad k \in \mathbb{N} .
$$

Similarly, take a deterministic digraph $\mathcal{G}^{\dagger}=\left(\mathrm{V}, \mathcal{E}^{\dagger}\right)$ with $\mathcal{E}^{\dagger}=\left\{\left(i_{\tau}, j_{\tau}\right): \tau=1, \ldots,\left|\mathcal{E}^{\dagger}\right|\right\}$. Denote

$$
\mathcal{E}_{k}(\tau):=\left\{\left(i_{\tau}, j_{\tau}\right) \in \mathrm{G}_{k}\right\}
$$

for $\tau=1, \ldots,\left|\mathcal{E}^{\dagger}\right|$ and $k \in \mathbb{N}$. We can now introduce the following concepts of $*$-mixing for the random graph process $\left\langle\mathrm{G}_{k}\right\rangle$.

Definition 6 A random graph process $\left\langle\mathrm{G}_{k}\right\rangle$ is termed

(i) connectivity $*$-mixing if $\left\langle\mathcal{C}_{k}\right\rangle$ is $*$-mixing;

(ii) arc $*$-mixing with respect to $\mathcal{G}^{\dagger}=\left(\mathrm{V}, \mathcal{E}^{\dagger}\right)$ if $\left\langle\mathcal{E}_{k}\left(\tau_{k}\right)\right\rangle$ is $*$-mixing for any deterministic sequence $\left\langle\tau_{k}\right\rangle$ with each $\tau_{k}$ taking value from $\left\{1, \ldots,\left|\mathcal{E}^{\dagger}\right|\right\}$.

\subsubsection{Consensus for $*$-Mixing Random Graphs}

We now present our main consensus convergence result for random graph processes that are connectivity or $\operatorname{arc} *$-mixing.

Theorem 4 Let $\left\langle\mathrm{G}_{k}\right\rangle$ be a random graph process equipped with a probability measure $\mathbb{P}$.

(i) Assume that $P_{k+1} \leq P_{k}$. Suppose there exist an integer $B \geq 1$ and a constant $0<q \leq 1$ such that

- $\langle\mathrm{G}([m B,(m+1) B-1])\rangle$ is connectivity $*$-mixing;

- $\mathbb{P}(\mathrm{G}([m B,(m+1) B-1])$ is quasi-strongly connected $) \geq q$ for all $k$.

Then Algorithm (1) achieves global a.s. consensus if $\sum_{s=0}^{\infty} P_{k}^{n-1}=\infty$.

(ii) Assume that $\left\langle\mathrm{G}_{k}\right\rangle$ is arc $*$-mixing with respect to $\mathcal{G}^{\dagger}$. Suppose there is a constant $0<q \leq 1$ such that $\mathbb{P}\left((i, j) \in \mathrm{G}_{k}\right) \geq q$ for all $(i, j) \in \mathcal{E}^{\dagger}$ and all $k \in \mathbb{N}$. Then Algorithm (1) achieves global a.s. consensus if and only if $\sum_{s=0}^{\infty} P_{k}=\infty$.

\footnotetext{
${ }^{4}$ There are various types of mixing $[47$. The $*$-mixing originated in $[4]$ we quote in this paper corresponds to the $\psi$-mixing in 47 .
} 
This theorem is proved following the same lines as the proofs of Proposition 11 and Theorem 3 for connectivity-independent and arc-independent graphs, where in the argument whenever necessary, we replace the standard Borel-Cantelli Lemma for a sequence of independent events with the corresponding Borel-Cantelli Lemma for $*$-mixing sequence of events established in [46]. The proof of Theorem 4 is in Appendix B.

On the other hand, the bounds of $\mathscr{T}_{\text {com }}(\epsilon)$ established in Proposition 1 and Theorem 3 no longer apply to the $*$-mixing random graphs. The reason is that the recursion of $\mathbf{E}[\mathcal{H}(k)]$ with arc/connectivity independence fails to stand under *-mixing, and therefore we cannot obtain the bounds of $\mathscr{T}_{\text {com }}(\epsilon)$ from the Markov's inequality. We however would like to point out that making use of the Strong Law of Large Numbers for $*$-mixing sequence of random variables (cf., Theorem 1, [46]), one can still build upper bounds for $\mathscr{T}_{\text {com }}(\epsilon)$ under the $*$-mixing conditions in Theorem 4, Apparently in this case $\mathscr{T}_{\text {com }}(\epsilon)$ relies not only on the presence of $*$-mixing, but also on the speed of $*$-mixing, i.e., the $f_{m}$ in Definition 5 ,

\subsubsection{An Illustrative Example}

It is known that for a strictly stationary, finite-state Markov chain, it is $*$-mixing if and only if it is irreducible and aperiodic (cf., Theorem 3.1, [47]). Then it becomes immediate that Theorem 4 is applicable to a large class of Markovian random graph processes $\left\langle\mathrm{G}_{k}\right\rangle$. We give an example below.

Example 5 [Random Walk on Graphs]. We define the random graph process $\left\langle\mathrm{G}_{k}\right\rangle$ as follows. Let $\mathcal{G}^{\mathrm{I}}=$ $\left(\mathrm{V}, \mathcal{E}^{\mathrm{I}}\right)$ be a strongly connected digraph for node interactions. Let $\left\langle s_{k}\right\rangle$ be an irreducible and aperiodic Markov chain with state space $\mathrm{V}$ and state transition matrix $P=\left[P_{i j}\right]$. Then $\left\langle s_{k}\right\rangle$ gives a random walk on the graph $\mathcal{G}_{P}$, where $\mathcal{G}_{P}$ is the induced graph of matrix $P$. The random graph $\mathrm{G}_{k}$ is defined as $\mathrm{E}_{k}=\left\{j:(j, i) \in \mathcal{E}^{\mathrm{I}}\right\}$ if $s_{k}=i$. Algorithms of this type have been used in networked sub-gradient algorithms for convex optimization [19].

Let $\left\langle s_{k}\right\rangle$ be initialized at its invariant distribution and take $B_{0}=\left|\mathcal{E}^{\mathrm{I}}\right|$. It is then straightforward to conclude that $\left\langle\mathrm{G}\left(\left[m B_{0},(m+1) B_{0}-1\right]\right)\right\rangle$ is connectivity $*$-mixing and there exists $q_{0}>0$ such that $\mathbb{P}(\mathrm{G}([m B,(m+1) B-1])$ is quasi-strongly connected $) \geq q_{0}$ for all $k$. Moreover, $\left\langle\mathrm{G}\left(\left[m B_{0},(m+1) B_{0}-1\right]\right)\right\rangle$ is also arc $*$-mixing with respect to $\mathcal{G}^{\mathrm{I}}$ and there also exists $q_{0}^{\prime}>0$ such that $\mathbb{P}((i, j) \in \mathrm{E}([m B,(m+1) B-$ $1])) \geq q_{0}^{\prime}$ for all $(i, j) \in \mathcal{E}^{\mathrm{I}}$ and for all $k$. Therefore, Theorem 4 is directly applicable.

\subsection{Random Gossiping}

Random gossiping is an important model in engineering (computer-to-computer, wireless communication) [2] and social networks [10]. The applicability of the established results to random gossiping algorithms has been discussed in Example 4. In standard random gossip algorithms, interactions always happen pairwise 
and the two interacting nodes average their states during their interaction. This simple nature of random gossiping certainly admits deeper results beyond the previous discussions. In this subsection, we make a further investigation to random gossiping algorithms.

\subsubsection{Gossiping Algorithms}

Let $S=\left[S_{i j}\right]$ be an $n \times n$ stochastic matrix. A gossip algorithm is given by a node pair selection process [2, 10].

Definition 7 Independently at each time $k \geq 0$, (i) a node $i \in \mathrm{V}$ is drawn with probability $1 / n$; (ii) the selected node $i$ picks the pair $\{i, j\}$ with probability $S_{i j}$.

This random pair selection process generates a sequence of i.i.d. random graphs $\left\langle\mathrm{G}_{k}\right\rangle$, where

$$
\mathrm{E}_{k}=\{(i, j),(j, i)\}
$$

when node pair $\{i, j\}$ is selected. When $\mathrm{E}_{k}=\{(i, j),(j, i)\}$, Algorithm (1) under this gossiping interaction reads as

(i) $x_{i}(k+1)=x_{i}(k) / 2+x_{j}(k) / 2$ with probability $P_{k}$, and $x_{i}(k+1)=x_{i}(k)$ with probability $1-P_{k}$;

(ii) Independent with node $i, x_{j}(k+1)=x_{i}(k) / 2+x_{j}(k) / 2$ with probability $P_{k}$, and $x_{j}(k+1)=x_{j}(k)$ with probability $1-P_{k}$;

(iii) $x_{j}(k+1)=x_{j}(k)$ for all $j \notin\{i, j\}$.

This model generalizes the standard gossiping algorithm in [2] with $\left\langle P_{k}\right\rangle$ characterizing possible communication failure in every gossiping pairs. Let the induced graph of $S, \mathcal{G}_{S}$, be quasi-strongly connected. We can apply Theorem 3 and directly conclude that if and only if $\sum_{k=0}^{\infty} P_{k}=\infty$, the above random gossiping algorithm converges almost surely. On the other hand, considering the following random graph process

$$
\left\langle\mathrm{G}\left(\left[m\left|\mathcal{E}_{S}\right|,(m+1)\left|\mathcal{E}_{S}\right|-1\right]\right)\right\rangle
$$

there holds

$$
\mathbf{P}\left(\mathrm{G}\left(\left[m\left|\mathcal{E}_{S}\right|,(m+1)\left|\mathcal{E}_{S}\right|-1\right]\right) \text { is quasi-strongly connected }\right) \geq\left(S_{*} / n\right)^{\left|\mathcal{E}_{S}\right|}
$$

with $S_{*}=\min \left\{S_{i j}+S_{j i}: S_{i j}+S_{j i}>0\right\}$. Therefore, Theorem 2 is also applicable to random gossiping process.

This structure of $\mathrm{E}_{k}$ in this random gossiping process however allows us to go beyond the asymptotic consensus analysis. We define a.s. finite-time consensus in the following. 
Definition 8 Global finite-time a.s. consensus is achieved for Algorithm (1) if for any initial value $x^{0}$, there exits a random time $K_{x^{0}}(\omega) \in \mathbb{N}$ which is a.s. finite such that $\mathbf{P}_{x^{0}}\left(\mathcal{H}\left(K_{x^{0}}\right)=0\right)=1$. The finite-time computation time $\mathscr{T}_{\text {com }}^{\mathrm{f}}$, is defined as

$$
\mathscr{T}_{\text {com }}^{\mathrm{f}}=\sup _{x^{0} \in \mathbb{R}^{n}} \min _{k}\{k \in \mathbb{N}: \mathcal{H}(k)=0\} .
$$

Note that $\mathscr{T}_{\text {com }}^{\mathrm{f}}$ is by definition a random variable, in contrast to $\mathscr{T}_{\text {com }}(\epsilon)$ which is a constant.

\subsubsection{Almost Sure Finite-time Convergence}

For finite-time convergence of random gossiping process, we present the following result.

Theorem 5 Let $\left\langle\mathrm{G}_{k}\right\rangle$ be a random gossiping process defined by stochastic matrix $S=\left[S_{i j}\right]$. Suppose $\mathcal{G}_{S}=\mathrm{K}_{n}$. Suppose $n=2^{m}+r$ with $0 \leq r<2^{m}$.

(i). Let $0<P_{*}<1 / 2$ be a constant such that $P_{*} \leq P_{k} \leq 1-P_{*}$ for all $k$ and suppose $\left\langle P_{k}\right\rangle$ is monotonic. Then global a.s. finite-time consensus is achieved if $\sum_{k=0}^{\infty}\left[P_{k}\left(1-P_{k}\right)\right]^{2 N_{0}}=\infty$ with $N_{0}=r+m(n+r) / 2$. Moreover, in this case there holds

$$
\mathbf{E}\left[\mathscr{T}_{\text {com }}^{\mathrm{f}}\right] \leq N_{0}\left(\frac{n}{P_{*}^{2} S_{*}}\right)^{N_{0}} .
$$

(ii). Let $P_{k}=1$ for all $k$. Then global a.s. finite-time consensus is achieved if and only if there is an integer $m>0$ such that $n=2^{m}$. In fact,

a) When $n=2^{m}$, there holds

$$
\mathbf{E}\left[\mathscr{T}_{\text {com }}^{\mathrm{f}}\right] \leq N\left(n / S_{*}\right)^{N}
$$

with $N=\left(n \log _{2} n\right) / 2$;

b) When $n$ is not some power of two (i.e., $r>0)$, we have $\mathbf{P}_{x^{0}}(\mathcal{H}(k)>0, k \in \mathbb{N})=0$ for almost all $x^{0} \in \mathbb{R}^{n}$ under standard Lebesgue measure.

The proof of Theorem [5 is in Appendix C. The proof is obtained by incorporating the results on finitetime convergence of deterministic gossip algorithms [44] with the Borel-Cantelli Lemma for independent sequence of events. Therefore, Theorem 5 continues to hold for a large class of Markovian gossiping processes (e.g., in Example 5, node $i$ only selects a node $j$ from its neighbors for gossiping when $s_{k}=i$.), based on the same $*$-mixing analysis as Theorem 4 .

It is worth emphasizing from Theorem 5 that, interestingly enough, the random update process on one hand decelerates the asymptotic convergence of the gossiping algorithm, but on the other hand makes finite time convergence in general possible (cf., without random node updates, i.e., $P_{k}=1$ for all $k$, a.s. finite-time convergence exists only if $n$ is some power of two, which is a rather strong restriction to the network). 


\section{Conclusions}

This paper investigated standard consensus algorithms coupled with randomized individual node decisionmaking over stochastically time-varying graphs. Each node determined its update by a sequence of Bernoulli trials with time-varying probabilities. The central aim of this work was to investigate the relation between the required level of independence of the graph process and the overall convergence. We consequently introduced connectivity-independence and arc-independence for the random graph processes. In light of the Borel-Cantelli lemma, a universal impossibility theorem showed that a.s. consensus cannot be achieved unless the sum of the success probability sequence diverges. Then a series of sufficiency conditions were given for the network to reach a global a.s. consensus under various connectivity assumptions. We established sharp threshold of almost sure consensus obeying the zero-one law. Convergence rates are established by lower and upper bounds of the $\epsilon$-computation time. We also generalized the concepts of connectivity/arc independence to their analogues from the $*$-mixing point of view, which covers the majority of random graph models in the literature such as Erdős-Rényi, gossiping, random walk, and Markovian random graphs. Under the $*$-mixing setting, our convergence analysis continued to stand and almost sure consensus conditions were established. Finally, we further investigated almost sure finite-time convergence of random gossiping algorithms. Surprisingly, the random node update process plays a key role in making a.s. finite-time convergence possible.

\section{Appendices}

\section{Appendix A. Proof of Theorem 1}

The proof relies on a few preliminary lemmas. First of all, the following lemma is well-known (see Theorem 15.5, pp. 300, [49]).

Lemma 7 Suppose $0 \leq b_{k}<1$ for all $k$. Then $\sum_{k=0}^{\infty} b_{k}=\infty$ if and only if $\prod_{k=0}^{\infty}\left(1-b_{k}\right)=0$.

Lemma 8 Suppose there exists a constant $a_{*}$ such that $a_{i i}(k) \geq a_{*}>1 / 2$ for all $i$ and $k$. Then for all $k \geq 0$, there always holds $\mathcal{H}(k+1) \geq\left(2 a_{*}-1\right) \mathcal{H}(k)$.

Proof. Let $x_{m}(k)=h(k)$ for some $m \in \mathrm{V}$. Then we have

$$
\begin{aligned}
\sum_{j \in \mathrm{N}_{m}(k)} a_{m j}(k) x_{j}(k) & =a_{m m}(k) x_{m}(k)+\sum_{j \in \mathrm{N}_{m}(k) \backslash\{m\}} a_{m j}(k) x_{j}(k) \\
& \leq a_{m m}(k) h(k)+\left(1-a_{m m}(k)\right) H(k) \\
& \leq a_{*} h(k)+\left(1-a_{*}\right) H(k)
\end{aligned}
$$


which implies

$$
h(k+1) \leq a_{*} h(k)+\left(1-a_{*}\right) H(k) .
$$

A symmetric argument leads to

$$
H(k+1) \geq\left(1-a_{*}\right) h(k)+a_{*} H(k)
$$

Based on (31) and (32), we obtain

$$
\begin{aligned}
\mathcal{H}(k+1) & =H(k+1)-h(k+1) \\
& \geq\left(1-a_{*}\right) h(k)+a_{*} H(k)-\left[a_{*} h(k)+\left(1-a_{*}\right) H(k)\right] \\
& \geq\left(2 a_{*}-1\right) \mathcal{H}(k) .
\end{aligned}
$$

The desired conclusion follows.

Let $\mathscr{S}_{0}$ be a set of stochastic matrices. Consider the following (deterministic) distributed averaging algorithms

$$
x(k+1)=W_{k} x(k),
$$

where each $W_{k} \in \mathscr{S}_{0}$ for all $k$. We define

$$
\mathscr{Z}_{0} \doteq\left\{z \in \mathbb{R}^{n}: \exists W_{0}, \ldots, W_{s} \in \mathscr{S}_{0}, s \geq 0 \text { s.t. } W_{s} \cdots W_{0} z \in \operatorname{span}\{\mathbf{1}\}\right\}
$$

Let $\mu(\cdot)$ represent the standard Lebesgue measure on $\mathbb{R}^{n}$. The following lemma from [44] holds for the finite-time convergence of averaging algorithm (34).

Lemma 9 Suppose $\mathscr{S}_{0}$ is a set with at most countable elements. Then either $\mathscr{Z}_{0}=\mathbb{R}^{n}$ or $\mu\left(\mathscr{Z}_{0}\right)=0$. In fact, if $\mathscr{Z}_{0} \neq \mathbb{R}^{n}$, then $\mathscr{Z}_{0}$ is a union of at most countably many linear spaces whose dimensions are no larger than $n-1$.

We are now ready to present the detailed proof of Theorem 1 .

Proof of (i). Let $\mathcal{H}(0)>0$. Then there exist at least two nodes, say $i$ and $j$ with different initial conditions. Considering the probability that both node $i$ and node $j$ remain constant: From Algorithm (1), we have

$$
\mathbf{P}\left(x_{i}(k+1)=x_{i}(k), \text { and } x_{j}(k+1)=x_{j}(k) k=0,1, \ldots\right) \geq\left[\prod_{k=0}^{\infty}\left(1-P_{k}\right)\right]^{2} \doteq r_{0}^{2},
$$

if $\sum_{k=0}^{\infty} P_{k}<\infty$, where $0<r_{0}=\prod_{k=0}^{\infty}\left(1-P_{k}\right)<1$ according to Lemma 7 . The necessity claim holds.

Next, let $\mathcal{H}(0)>0$ and denote by $i$ and $j$ two nodes satisfying $x_{i}(0)=h(0)$ and $x_{j}(0)=H(0)$, respectively. Notice that when both of them never make a state update, they will remain at all times 
with minimum (resp. maximum) states, because the other nodes cannot go further by making convex combinations. Hence,

$$
\mathbb{P}(\mathcal{H}(k)>\epsilon \mathcal{H}(0)) \geq P(\mathcal{H}(k) \geq \mathcal{H}(0)) \geq \prod_{h=0}^{k-1}\left(1-P_{h}\right)^{2} .
$$

Then the lower bound of the $\epsilon$-computation can be easily obtained.

Proof of (ii). We rewrite Algorithm (10) into

$$
x(k+1)=A_{k} x_{k}
$$

with $A_{k}=\left[a_{i j}(k)\right]$ being a random matrix taking value from the set of stochastic matrices. Now that $\left\{a_{i j}(k): i, j \in \mathrm{V}, k \in \mathbb{N}\right\}$ contains at most countably many elements, applying Lemma 9 we conclude that one of the following two cases must happen:

$\left.a^{\dagger}\right)$ There exists a constant $K \geq 0$ under which a sample path $A_{k}(\omega)$ satisfies

$$
\operatorname{rank}\left(A_{K}(\omega) \ldots A_{0}(\omega)\right)=1
$$

This implies that $\mathcal{H}(K)(\omega)=0$ for all initial value $x^{0}$.

$\left.b^{\dagger}\right)$ For almost all initial value $x^{0}$ and all $\omega, \mathcal{H}(k)(\omega)>0$ for all $k \in \mathbb{N}$.

The First Borel-Cantelli Lemma [35] ensures that every node updates its state only for some finite times when $\sum_{k=0}^{\infty} P_{k}<\infty$. Therefore, it becomes immediate to see that the cases $\left.a^{\dagger}\right)$ and $b^{\dagger}$ ) correspond to cases $a$ ) and $b$ ), respectively.

Proof of (iii). Let $\mathcal{H}(0)>0$. There holds

$$
\sum_{k=0}^{\infty} P_{k}<\infty \Leftrightarrow \prod_{k=0}^{\infty}\left(1-P_{k}\right)>0 \Leftrightarrow \prod_{k=0}^{\infty}\left(1-P_{k}\right)^{n}>0 \Leftrightarrow \sum_{k=0}^{\infty}\left(1-\left(1-P_{k}\right)^{n}\right)<\infty,
$$

where the last equivalence is obtained by taking $b_{k}=1-\left(1-P_{k}\right)^{n}$ in Lemma 7. From the definition of Algorithm (1) we see that

$$
\mathbf{P}(\mathcal{H}(k+1)<\mathcal{H}(k)) \leq \mathbf{P}(\text { at least one node takes averaging at time } k)=1-\left(1-P_{k}\right)^{n} .
$$

In light of Lemma 8, and applying the First Borel-Cantelli Lemma [35] on (37), it follows immediately that

$$
\mathbf{P}\left(\lim _{k \rightarrow \infty} \mathcal{H}(k)=0\right) \leq \mathbf{P}(\mathcal{H}(k+1)<\mathcal{H}(k) \text { for infinitely many } k)=0
$$

The desired conclusion follows.

We have now completed the proof. 


\section{Appendix B. Proof of Theorem 4}

The proof is based on the following Borel-Cantelli lemma for $*$-mixing sequence of events.

Lemma 10 (Lemma 6, [46]) Let $\left\langle\mathcal{A}_{k}\right\rangle$ be a sequence of events which is $*$-mixing. Then $\sum_{k=0}^{\infty} \mathbb{P}\left(\mathcal{A}_{k}\right)=\infty$ implies $\mathbb{P}\left(\lim \sup _{k \rightarrow \infty} \mathcal{A}_{k}\right)=1$.

(i). Let $k_{s}=s(n-1)^{2} B$. We introduce the following sequence of events $\left\langle\mathfrak{E}_{s}\right\rangle$ :

$$
\mathcal{H}_{s}:=\left\{\mathcal{H}\left(k_{s+1}\right) \leq\left(1-\frac{\eta^{(n-1)^{2}}}{2}\right) \mathcal{H}\left(k_{s}\right)\right\}, s=0,1, \ldots
$$

We also define the sequence of event $\left\langle\mathcal{B}_{m}\right\rangle$ :

$$
\mathcal{B}_{m}:=\{\mathrm{G}([m B,(m+1) B-1]) \text { is quasi-strongly connected }\}, m=0,1, \ldots
$$

The proof of Proposition 1 implies

$$
\mathcal{H}_{s} \supseteq Z_{s}
$$

where by definition

$$
Z_{s}:=\left(\bigcap_{m=0}^{(n-1)^{2} B-1} \mathcal{B}_{s(n-1)^{2} B+m}\right) \cap\left\{\text { the } i_{1}, \ldots, i_{n-1} \in \mathrm{V} \text { in turn update their states }\right\} .
$$

Noticing the facts that $\left\langle\mathcal{B}_{m}\right\rangle$ is $*$-mixing, and the node updates are independent of the graph process, node states and other nodes, $\left\langle Z_{s}\right\rangle$ is also $*$-mixing. Moreover, with the monotonicity of $\left\langle P_{k}\right\rangle$, there holds

$$
\mathbf{P}\left(Z_{s}\right) \geq(q / n)^{(n-1)^{2}} P_{s(n-1)^{2} B}^{n-1}
$$

from the same argument as we obtain (9).

Therefore, we can apply Lemma 10 to $\left\langle Z_{s}\right\rangle$, and conclude from (39) and (40) that if $\sum_{k=0}^{\infty} P_{k}^{n-1}=\infty$, then

$$
\mathbf{P}\left(\limsup _{s \rightarrow \infty} \mathcal{H}_{s}\right) \geq \mathbf{P}\left(\limsup _{s \rightarrow \infty} Z_{s}\right)=1 .
$$

Observing that the event $\lim \sup _{s \rightarrow \infty} \mathcal{H}_{s}$ means the $\mathcal{H}_{s}$ happen for infinitely many $s$, and that $\mathcal{H}(k+1) \leq$ $\mathcal{H}(k)$ always holds true, (41) immediately leads to global a.s. consensus for Algorithm (11). We have now completed the proof for connectivity $*$-mixing random graphs.

(ii). Again, the proof is obtained by adapting the proof of Theorem 3 to the case of arc $*$-mixing graphs. All we need is to adopting the above Borel-Cantelli argument after (25), instead of the original one using Fatou's Lemma. The details are therefore omitted.

We have now completed the proof. 


\section{Appendix C. Proof of Theorem 5}

The proof is built upon some preliminary results on deterministic finite-time gossiping [44] (for a more comprehensive treatment we hereby refer to [45]). Introduce the following two sets of stochastic matrices:

$$
\mathfrak{M}_{n}:=\left\{I_{n}-\frac{\left(e_{i}-e_{j}\right)\left(e_{i}-e_{j}\right)^{T}}{2}: i, j \in \mathrm{V}\right\} ; \mathfrak{M}_{n}^{\dagger}:=\left\{I_{n}-\frac{e_{i}\left(e_{i}-e_{j}\right)^{T}}{2}: i, j \in \mathrm{V}\right\} .
$$

The matrices in $\mathfrak{M}_{n}$ and $\mathfrak{M}_{n}^{\dagger}$ represent the network state transition matrix for the realizations of successful node pair interactions [2].

(i). We recall the following conclusion established in [44].

Lemma 11 Suppose $n=2^{m}+r$ with $m \geq 0$ and $0 \leq r<2^{m}$. Then there are $N_{0}=r+m(n+r) / 2$ matrices $M_{1}, \cdots, M_{N_{0}} \in \mathfrak{M}_{n} \bigcup \mathfrak{M}_{n}^{\dagger}$ such that $\operatorname{rank}\left(M_{N_{0}} \ldots M_{1}\right)=1$.

Now let $M_{1}, \ldots, M_{N_{0}}$ be the $N_{0}$ matrices defined in Lemma 11 from the set $\mathfrak{M}_{n} \bigcup \mathfrak{M}_{n}^{\dagger}$ with

$$
\operatorname{rank}\left(M_{N_{0}} \ldots M_{1}\right)=1
$$

Each $M_{k}$ corresponds to a pair of nodes $\left\{i_{k}, j_{k}\right\}$. The probability of a given pair of nodes selected at time $k$ is no smaller than $S_{*} / n$ from the definition of the gossiping process. Depending on $M_{k} \in \mathfrak{M}_{n}$ or $M_{k} \in \mathfrak{M}_{n}^{\dagger}$, there are two cases: $M_{k}$ is realized when both the two nodes $\left\{i_{k}, j_{k}\right\}$ successfully update their states; $M_{k}$ is realized when only one node $\left(i_{k}\right.$ or $\left.j_{k}\right)$ successfully updates its state. For either of the two cases we call the selected node pair $\left\{i_{k}, j_{k}\right\}$ realizes $M_{k}$. Then at time $k, M_{k}$ is realized with probability either $P_{k}^{2}$ or $P_{k}\left(1-P_{k}\right)$ given that $\left\{i_{k}, j_{k}\right\}$ is selected.

Consider the following events:

$$
\mathcal{E}_{s}:=\left\{\left(i_{k}, j_{k}\right) \in \mathrm{E}_{s N_{0}+k-1} \text { and } M_{k} \text { is realized, } k=1, \ldots, N_{0}\right\}, \quad s \in \mathbb{N} .
$$

Note that $\operatorname{rank}\left(M_{N_{0}} \ldots M_{1}\right)$ implies that for all initial value $x^{0}$, any $\mathcal{E}_{s-1}$ happening will lead to $\mathcal{H}\left(s N_{0}\right)=$ 0.

Suppose that $\left\langle P_{k}\right\rangle$ is non-increasing and without loss of generality we let $P_{0}<1$. Then there holds for all $k$ that

$$
P_{k}\left(1-P_{k}\right) \geq\left(1-P_{0}\right) P_{k} \geq\left(1-P_{0}\right) P_{k}^{2},
$$

and $\sum_{k=0}^{\infty}\left[P_{k}\left(1-P_{k}\right)\right]^{2 N_{0}}=\infty$ leads to $\sum_{k=0}^{\infty} P_{k}^{2 N_{0}}=\infty$. This gives us

$$
\mathbf{P}\left(\mathcal{E}_{s-1}\right) \geq\left(\left(1-P_{0}\right)\left(S_{*} / n\right) P_{s N_{0}}^{2}\right)^{N_{0}}
$$

Thus, invoking the Borel-Cantelli lemma, a.s. finite-time consensus is achieved for Algorithm (5) if $\sum_{k=0}^{\infty}\left[P_{k}\left(1-P_{k}\right)\right]^{2 N_{0}}=\infty$. 
The case when $\left\langle P_{k}\right\rangle$ is non-decreasing holds from a symmetric argument, whose details are therefore omitted. This concludes the proof of a.s. finite-time convergence.

Next, letting $P_{*} \leq P_{k} \leq 1-P_{*}$ for all $k$, we establish the upper bound for $\mathbf{E}\left[\mathscr{T}_{\text {com }}^{\mathrm{f}}\right]$. Note that there always holds

$$
\mathscr{T}_{\text {com }}^{\mathrm{f}} \leq N_{0} \inf _{s}\left\{s \geq 1: \mathcal{E}_{s-1} \text { happens }\right\}
$$

As a result, we conclude that

$$
\mathbf{E}\left[\mathscr{T}_{\text {com }}^{\mathrm{f}}\right] \leq \sum_{k=1}^{\infty} N_{0} k(1-p)^{k-1} p=N_{0} \sum_{k=0}^{\infty}(1-p)^{k}=N_{0} / p,
$$

where $p=\left(P_{*}^{2} S_{*} / n\right)^{N_{0}}$ is a lower bound for the probability of $\mathcal{E}_{s}$. This proves the proposed upper bound for $\mathbf{E}\left[\mathscr{T}_{\text {com }}^{\mathrm{f}}\right]$.

(ii). The proof requires the following conclusion from [44].

Lemma 12 (i) If $n=2^{m}$, then there are $N=\frac{n \log _{2} n}{2}$ matrices $M_{1}, \ldots, M_{N}$ with each $M_{i} \in \mathfrak{M}_{n}$ such that $M_{N} \cdots M_{1}=11^{\mathrm{T}} / n$.

(ii) If $n$ is not some power of two, then for almost all initial value $x^{0}$, there holds

$$
M_{t} \cdots M_{1} x^{0} \notin \operatorname{span}\{\mathbf{1}\}
$$

for all $M_{1}, \ldots, M_{t} \in \mathfrak{M}_{n}$ and for all $t \in \mathbb{N}$.

Proof of a). Let $n=2^{m}$ for some integer $m$. Let $M_{1}, \ldots, M_{N}$ be the $N=\frac{n \log _{2} n}{2}$ matrices defined in Lemma 12 from the set $\mathfrak{M}_{n}$ with $M_{N} \cdots M_{1}=\mathbf{1 1}^{\mathrm{T}} / n$. Similarly, each $M_{k}$ corresponds to a pair of nodes $\left\{i_{k}, j_{k}\right\}$, and he probability of a given pair of nodes selected at time $k$ is no smaller than $S_{*} / n$ from the definition of the gossiping process. Consider the following events:

$$
\mathcal{D}_{s}:=\left\{\left(i_{k}, j_{k}\right) \in \mathrm{E}_{s N+k-1}, k=1, \ldots, N\right\}, \quad s \in \mathbb{N} .
$$

Since $P_{k}=1$ for all $k$, the fact that $M_{N} \cdots M_{1}=\mathbf{1 1}^{\mathrm{T}} / n$ implies that for all initial value $x^{0}$, any $\mathcal{D}_{s-1}$ happening will lead to $\mathcal{H}(s N)=0$. Therefore, a.s. finite-time consensus follows immediately from the Borel-Cantelli lemma. Moreover, there always holds

$$
\mathscr{T}_{\text {com }}^{\mathrm{f}} \leq N \inf _{s}\left\{s \geq 1: \mathcal{D}_{s-1} \text { happens }\right\}
$$

Similarly, with $p_{*}=\left(S_{*} / n\right)^{N}$ being a lower bound for the probability of $\mathcal{D}_{s}$, we obtain

$$
\mathbf{E}\left[\mathscr{T}_{\text {com }}^{\mathrm{f}}\right] \leq N / p_{*},
$$

This proves the proposed upper bound for $\mathbf{E}\left[\mathscr{T}_{\text {com }}^{\mathrm{f}}\right]$. 
Proof of b). The desired conclusion follows directly from Lemma 12,(ii), which indeed reveals the impossibility of finite-time consensus for every sample path for almost all initial values.

We have now completed the proof of the proposition.

\section{Acknowledgements}

This work has been supported in part by the Knut and Alice Wallenberg Foundation, the Swedish Research Council, KTH SRA TNG, and by NICTA Ltd and the Australian Research Council (ARC) under DP110100538 and DP-130103610. A brief version of the current manuscript was presented at the American Control Conference in Montreal, Canada, July 2012 [48].

\section{References}

[1] A. Jadbabaie, J. Lin, and A. S. Morse, "Coordination of groups of mobile autonomous agents using nearest neighbor rules," IEEE Trans. Autom.Control, vol. 48, no. 6, pp. 988-1001, 2003.

[2] S. Boyd, A. Ghosh, B. Prabhakar and D. Shah, "Randomized gossip algorithms," IEEE Trans. Inf. Theory, vol. 52, no. 6, pp. 2508-2530, 2006.

[3] D. Mosk-Aoyama and D. Shah, "Fast distributed algorithms for computing separable functions," IEEE Trans. Inf. Theory, vol.55, no.7, pp. 2997-3007, 2008

[4] C. C. Moallemi and B. Van Roy, "Consensus propagation," IEEE Trans. Inf. Theory, vol. 52, no. 11, pp. 4753-4766, 2006.

[5] S. Muthukrishnan, B. Ghosh, and M. Schultz, "First and second order diffusive methods for rapid, coarse, distributed load balancing," Theory of Computing Systems, vol. 31, pp. 331-354, 1998.

[6] R. Diekmann, A. Frommer, and B. Monien, "Efficient schemes for nearest neighbor load balancing," Parallel Computing, vol. 25, pp. 789-812, 1999.

[7] S. Martinez, J. Cortés, and F. Bullo, "Motion coordination with distributed information," IEEE Control Systems Magazine, vol. 27, no. 4, pp. 75-88, 2007.

[8] W. Ren and R. Beard, "Consensus seeking in multi-agent systems under dynamically changing interaction topologies," IEEE Trans. Autom. Control, vol. 50, no. 5, pp. 655-661, 2005. 
[9] M. H. DeGroot, "Reaching a consensus," Journal of the American Statistical Association, vol. 69, no. 345, pp. 118-121, 1974.

[10] D. Acemoglu, A. Ozdaglar and A. ParandehGheibi, "Spread of (Mis)information in social networks," Games and Economic Behavior, vol. 70, no. 2, pp. 194-227, 2010.

[11] D. Acemoglu, G. Como, F. Fagnani, A. Ozdaglar, "Opinion fluctuations and persistent disagreement in social networks," in IEEE Conference on Decision and Control, pp. 2347-2352, Orlando, 2011.

[12] R. Olfati-Saber and R. Murray, "Consensus problems in the networks of agents with switching topology and time dealys," IEEE Trans. Autom. Control, vol. 49, no. 9, pp. 1520-1533, 2004.

[13] J. Fax and R. Murray, "Information flow and cooperative control of vehicle formations," IEEE Trans. Autom. Control, vol. 49, no. 9, pp. 1465-1476, 2004.

[14] L. Moreau, "Stability of multi-agent systems with time-dependent communication links," IEEE Trans. Autom. Control, vol. 50, pp. 169-182, 2005.

[15] M. Cao, A. S. Morse and B. D. O. Anderson, "Reaching a consensus in a dynamically changing environment: a graphical approach," SIAM J. Control Optim., vol. 47, no. 2, 575-600, 2008.

[16] J. Hajnal, "Weak ergodicity in non-homogeneous markov chains," Proc. Cambridge Philos. Soc., no. 54, pp. 233-246, 1958.

[17] J. Wolfowitz, "Products of indecomposable, aperiodic, stochastic matrices," Proc. Amer. Math. Soc., vol. 15, pp. 733-736, 1963.

[18] J. N. Tsitsiklis. Problems in Decentralized Decision Making and Computation. Ph.D. thesis, Dept. of Electrical Engineering and Computer Science, Massachusetts Institute of Technology, Boston, MA, 1984.

[19] S. Boyd, P. Diaconis and L. Xiao, "Fastest mixing markov chain on a graph," SIAM Review, Vol. 46, No. 4, pp. 667-689, 2004.

[20] K. Jung, D. Shah, and J. Shin, "Distributed averaging via lifted Markov chains," IEEE Trans. Inf. Theory, vol. 56, no. 1, pp. 634-647, 2010.

[21] Y. Hatano and M. Mesbahi, "Agreement over random networks," IEEE Trans. Autom. Control, vol. 50, no. 11, pp. 1867-1872, 2005. 
[22] C. W. Wu, "Synchronization and convergence of linear dynamics in random directed networks," IEEE Trans. Autom. Control, vol. 51, no. 7, pp. 1207-1210, 2006.

[23] F. Fagnani and S. Zampieri, "Randomized consensus algorithms over large scale networks," IEEE J. on Selected Areas of Communications, vol. 26, no.4, pp. 634-649, 2008.

[24] F. Fagnani and S. Zampieri, "Average consensus with packet drop communication," SIAM J. Control Optim., vol. 48, no. 1, pp. 102-133, 2009.

[25] A. Tahbaz-Salehi and A. Jadbabaie, "A necessary and sufficient condition for consensus over random networks," IEEE Trans. Autom. Control, vol. 53, no. 3, pp. 791-795, 2008.

[26] U. A. Khan, S. Kar, and J. M. F. Moura, "Distributed sensor localization in random environments using minimal number of anchor nodes," IEEE Trans. Signal Processing, 57: 5, pp. 2000-2016, 2009.

[27] S. Kar and J. M. F. Moura, "Distributed consensus algorithms in sensor networks: quantized data and random link failures," IEEE Trans. Signal Processing, Vol. 58:3, pp. 1383-1400, 2010.

[28] S. Patterson, B. Bamieh and A. El Abbadi, "Convergence rates of distributed average consensus with stochastic link failures," IEEE Trans. Autom. Control, vol. 55, no. 4, pp. 880-892, 2010.

[29] I. Matei, N. Martins and J. S. Baras, "Almost sure convergence to consensus in Markovian random graphs," in Proc. IEEE Conf. Decision and Control, pp. 3535-3540, 2008.

[30] T. C. Aysal and K. E. Barner, "Convergence of consensus models with stochastic disturbances," IEEE Trans. Inf. Theory, vol. 56, no. 8, pp. 4101-4113, 2010.

[31] A. Tahbaz-Salehi and A. Jadbabaie, "Consensus over ergodic stationary graph processes," IEEE Trans. Autom. Control, vol. 55, no. 1, 2010.

[32] G. Latouche, V. Ramaswami. Introduction to Matrix Analytic Methods in Stochastic Modeling. 1st edition, ASA SIAM, 1999.

[33] C. Godsil and G. Royle. Algebraic Graph Theory. New York: Springer-Verlag, 2001.

[34] C. Berge and A. Ghouila-Houri. Programming, Games, and Transportation Networks. John Wiley and Sons, New York, 1965.

[35] R. Durrett. Probability: Theory and Examples. Duxbury advanced series, Third Edition, Thomson Brooks/Cole, 2005.

[36] B. Bollobás. Random Graphs. Cambridge University Press, second edition, 2001. 
[37] A. Ramamoorthy, A. J. Shi, and R. D. Wesel, "On the capacity of network coding for random networks," IEEE Trans. Inf. Theory, vol. 51 , no. 8, pp. 2878-2885, 2005.

[38] P. Erdős and A. Rényi, "On the evolution of random graphs," Publications of the Mathematical Institute of the Hungarian Academy of Sciences, 17-61, 1960.

[39] P. Gupta and P. R. Kumar, "The capacity of wireless networks," IEEE Trans. Inf. Theory, vol. 46, no. 2, pp.388-404, 2000.

[40] T. K. Philips, D. F. Towsley and J. K. Wolf, "On the diameter of a class of random graphs," IEEE Trans. Inf. Theory, vol. 36, no. 2, pp. 285-288, 1990.

[41] F. Xue and P. R. Kumar, "On the $\theta$-coverage and connectivity of large random networks," IEEE Trans. Inf. Theory, vol. 52, no. 6, pp. 2289-2299, 2006.

[42] O. Yagan and A. M. Makowski, "Zero-one laws for connectivity in random key graphs," IEEE Trans. Inf. Theory, vol. 58 , no. 5 , pp. 2983-2999, 2012.

[43] A. Nedić, A. Olshevsky, A. Ozdaglar, and J. N. Tsitsiklis, "On distributed averaging algorithms and qantization effects," IEEE Trans. Autom. Control, vol. 54, no. 11, pp. 2506-2517, 2009.

[44] G. Shi, B. Li, M. Johansson and K. H. Johansson, "When do gossip algorithms converge in finite time?" in The 21st International Symposium on Mathematical Theory of Networks and Systems (MTNS), Groningen, The Netherlands, Jul. 2014.

[45] G. Shi, B. Li, M. Johansson and K. H. Johansson, "Finite-time convergent gossiping," preprint, avaiable at arXiv:1206.0992, 2015.

[46] J. R. Blum, D. L. Hanson, and L. H. Koopmans, "On the strong law of large numbers for a class of stochastic processes," Z. Wahrsch. verw. Gebiete, vol. 2 pp. 1-11, 1963.

[47] R. C. Bradley, "Basic properties of strong mixing conditions: a survey and some open questions," Probability Surveys, vol. 2, pp. 107-144, 2005.

[48] G. Shi and K. H. Johansson, "Agreeing under randomized network dynamics," American Control Conference, pp. 2394-2400, Montreal, Canada, Jun. 2012.

[49] W. Rudin. Real and Complex Analysis. Third Edition, McGraw-Hill, 1987.

[50] E. Seneta. Non-negative Matrices and Markov Chains. Allen \& Unwin Ltd., London, 1973. 


\section{Guodong Shi and Brian D. O. Anderson}

Research School of Engineering, College of Engineering and Computer Science,

The Australian National University, Canberra, ACT 0200, Australia.

Email: guodong.shi@anu.edu.au, brian.anderson@anu.edu.au

KARL H. JOHANSSON

ACCESS Linnaeus Centre, School of Electrical Engineering,

KTH Royal Institute of Technology, Stockholm 100 44, Sweden.

Email: kallej@kth.se 